\title{
Esophageal and Esophagogastric Junction Cancers, Version 2.2019
}

Jaffer A. Ajani, MD,*; Thomas A. D'Amico, MD²; David J. Bentrem, MD, MS; Joseph Chao, MD; Carlos Corvera, MD; Prajnan Das, MD, MS, MPH${ }^{1}$; Crystal S. Denlinger, MD ; Peter C. Enzinger, MD; Paul Fanta, MD; Farhood Farjah, MD,*; Hans Gerdes, MD ${ }^{10}$; Michael Gibson, MD, PhD ${ }^{11}$; Robert E. Glasgow, MD ${ }^{12}$; James A. Hayman, MD, MBA ${ }^{13}$; Steven Hochwald, MD ${ }^{14}$; Wayne L. Hofstetter, MD'; David H. Ilson, MD, PhD ${ }^{10, *}$; Dawn Jaroszewski, MD ${ }^{15}$; Kimberly L. Johung, MD, PhD ${ }^{16, *}$; Rajesh N. Keswani, MD ${ }^{3, *}$; Lawrence R. Kleinberg, MD ${ }^{17}$; Stephen Leong, MD ${ }^{18}$; Quan P. Ly, MD ${ }^{19} ;$ Kristina A. Matkowskyj, MD, PhD 20,*; Michael McNamara, MD²1; Mary F. Mulcahy, MD³; Ravi K. Paluri, MD, MPH'22; Haeseong Park, MD, MPH ${ }^{23}$; Kyle A. Perry, MD²4; Jose Pimiento, MD ${ }^{25, *}$; George A. Poultsides, MD, MS ${ }^{26}$; Robert Roses, MD²7,*; Vivian E. Strong, MD ${ }^{10}$; Georgia Wiesner, MD ${ }^{11}$;

Christopher G. Willett, MD²; Cameron D. Wright, MD ${ }^{28}$; Nicole R. McMillian, MS, CHES ${ }^{29}$; and Lenora A. Pluchino, PhD ${ }^{29}$

\section{ABSTRACT}

Esophageal cancer is the sixth leading cause of cancer-related deaths worldwide. Squamous cell carcinoma is the most common histology in Eastern Europe and Asia, and adenocarcinoma is most common in North America and Western Europe. Surgery is a major component of treatment of locally advanced resectable esophageal and esophagogastric junction (EGJ) cancer, and randomized trials have shown that the addition of preoperative chemoradiation or perioperative chemotherapy to surgery significantly improves survival. Targeted therapies including trastuzumab, ramucirumab, and pembrolizumab have produced encouraging results in the treatment of patients with advanced or metastatic disease. Multidisciplinary team management is essential for all patients with esophageal and EGJ cancers. This selection from the NCCN Guidelines for Esophageal and Esophagogastric Junction Cancers focuses on recommendations for the management of locally advanced and metastatic adenocarcinoma of the esophagus and EGJ.

J Natl Compr Canc Netw 2019;17(7):855-883 doi: $10.6004 /$ jnccn.2019.0033

\footnotetext{
${ }^{1}$ The University of Texas MD Anderson Cancer Center; ${ }^{2}$ Duke Cancer Institute; ${ }^{3}$ Robert H. Lurie Comprehensive Cancer Center of Northwestern University; ${ }^{4}$ City of Hope National Medical Center; ${ }^{5}$ UCSF Helen Diller Family Comprehensive Cancer Center; ${ }^{6}$ Fox Chase Cancer Center; ${ }^{7}$ Dana-Farber/ Brigham and Women's Cancer Center; ${ }^{8}$ UC San Diego Moores Cancer Center; ${ }^{9}$ Fred Hutchinson Cancer Research Center/Seattle Cancer Care Alliance;

${ }^{10}$ Memorial Sloan Kettering Cancer Center; ${ }^{11}$ Vanderbilt-Ingram Cancer Center; ${ }^{12}$ Huntsman Cancer Institute at the University of Utah; ${ }^{13}$ University of Michigan Rogel Cancer Center; ${ }^{14}$ Roswell Park Comprehensive Cancer Center; ${ }^{15}$ Mayo Clinic Cancer Center; ${ }^{16}$ Yale Cancer Center/Smilow Cancer Hospital; ${ }^{17}$ The Sidney Kimmel Comprehensive Cancer Center at Johns Hopkins; ${ }^{18}$ University of Colorado Cancer Center; ${ }^{19}$ Fred \& Pamela Buffett Cancer Center; ${ }^{20}$ University of Wisconsin Carbone Cancer Center; ${ }^{21}$ Case Comprehensive Cancer Center/ University Hospitals Seidman Cancer Center and Cleveland Clinic Taussig Cancer Institute; ${ }^{22} \mathrm{O}^{\prime} \mathrm{Neal}$ Comprehensive Cancer Center at UAB; ${ }^{23}$ Siteman Cancer Center at Barnes-Jewish Hospital and Washington University School of Medicine; ${ }^{24}$ The Ohio State University Comprehensive Cancer Center - James Cancer Hospital and Solove Research Institute; ${ }^{25}$ Moffitt Cancer Center;

${ }^{26}$ Stanford Cancer Institute; ${ }^{27}$ Abramson Cancer Center at the University of Pennsylvania; ${ }^{28}$ Massachusetts General Hospital Cancer Center; and ${ }^{29}$ National Comprehensive Cancer Network
}

*Discussion Writing Committee Member

\section{NCCN CATEGORIES OF EVIDENCE AND CONSENSUS}

Category 1: Based upon high-level evidence, there is uniform NCCN consensus that the intervention is appropriate.

Category 2A: Based upon lower-level evidence, there is uniform NCCN consensus that the intervention is appropriate.

Category 2B: Based upon lower-level evidence, there is NCCN consensus that the intervention is appropriate.

Category 3: Based upon any level of evidence, there is major NCCN disagreement that the intervention is appropriate.

All recommendations are category 2A unless otherwise noted.

Clinical trials: NCCN believes that the best management of any patient with cancer is in a clinical trial. Participation in clinical trials is especially encouraged.

\section{PLEASE NOTE}

The NCCN Clinical Practice Guidelines in Oncology (NCCN Guidelines ${ }^{\circledR}$ ) are a statement of evidence and consensus of the authors regarding their views of currently accepted approaches to treatment. Any clinician seeking to apply or consult the NCCN Guidelines is expected to use independent medical judgment in the context of individual clinical circumstances to determine any patient's care or treatment. The National Comprehensive Cancer Network ${ }^{\circledR}\left(\mathrm{NCCN}^{\circledR}\right)$ makes no representations or warranties of any kind regarding their content, use, or application and disclaims any responsibility for their application or use in any way.

The complete NCCN Guidelines for Esophageal and Esophagogastric Junction Cancers are not printed in this issue of JNCCN but can be accessed online at NCCN.org.

(C) National Comprehensive Cancer Network, Inc. 2019. All rights reserved. The NCCN Guidelines and the illustrations herein may not be reproduced in any form without the express written permission of $\mathrm{NCCN}$.

\section{Disclosures for the NCCN Esophageal and Esophagogastric Junction Cancers Panel}

At the beginning of each NCCN Guidelines Panel meeting, panel members review all potential conflicts of interest. NCCN, in keeping with its commitment to public transparency, publishes these disclosures for panel members, staff, and NCCN itself. Individual disclosures for the NCCN Esophageal and Esophagogastric Junction Cancers Panel members can be found on page 883. (The most recent version of these guidelines and accompanying disclosures are available at NCCN.org.)

The complete and most recent version of these guidelines is available free of charge at NCCN.org. 


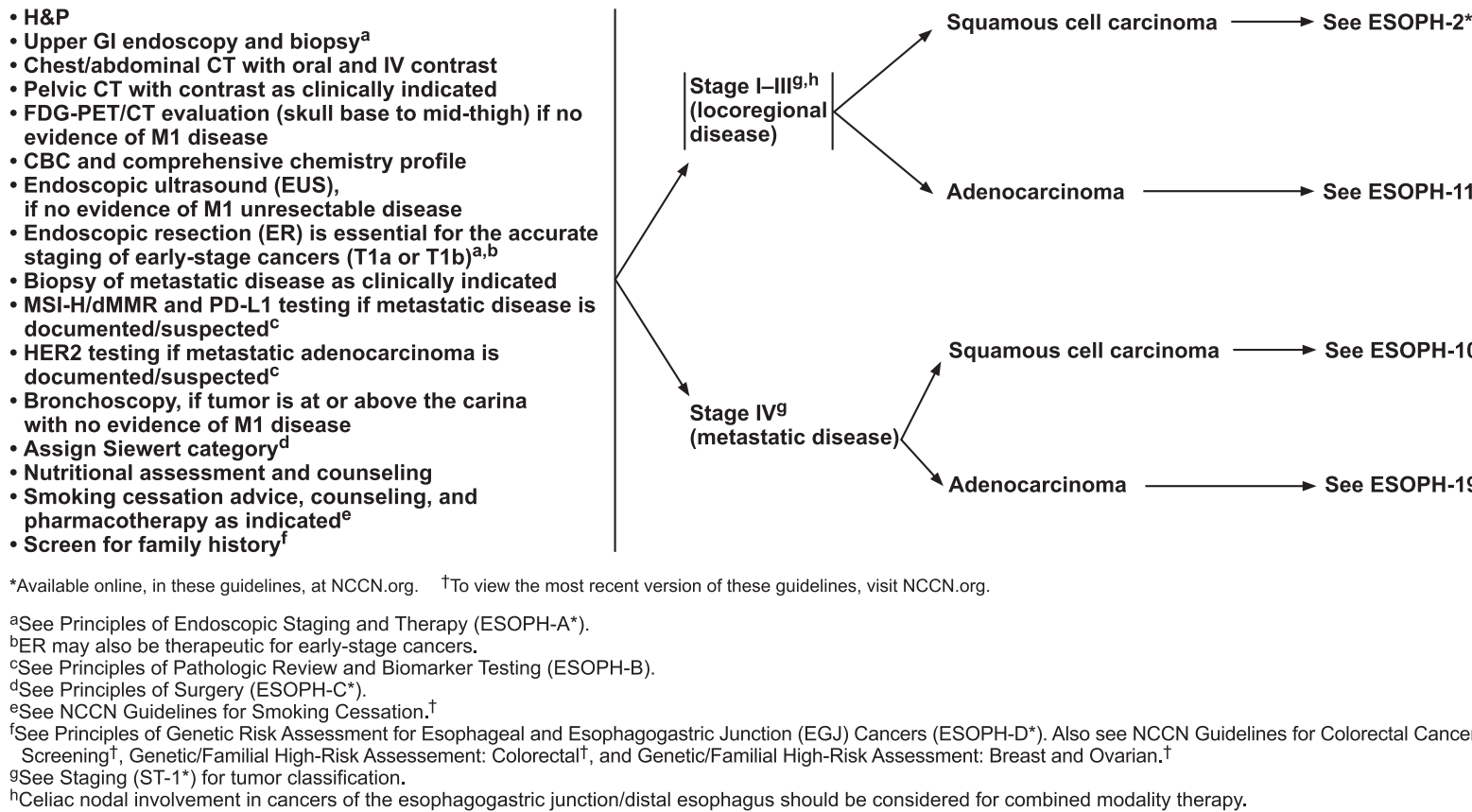

\section{Overview}

Upper gastrointestinal (GI) tract cancers originating in the esophagus and esophagogastric junction (EGJ) constitute a major global health problem, especially in low and middle income countries. ${ }^{1}$ Globally, there were an estimated 572,000 cases resulting in more than 508,000 deaths in 2018, making esophageal cancer the seventh most frequently diagnosed cancer and the sixth leading cause of cancer-related deaths in the world. ${ }^{2,3}$ In contrast, esophageal cancer is one of the least commonly diagnosed cancers in North America. In 2019, an estimated 17,650 people will be diagnosed and 16,080 people will die of this disease in the United States, making esophageal cancer the 20th most commonly diagnosed cancer and the 11th leading cause of cancer-related death in America. ${ }^{4,5}$

Esophageal cancers are histologically classified as squamous cell carcinoma (SCC) or adenocarcinoma, which differ in their pathology, tumor location, and prognosis. ${ }^{6}$ In contrast to adenocarcinoma, SCC is more likely to localize near the tracheal bifurcation, has a proclivity for earlier lymphatic spread, and is associated with a poorer prognosis. ${ }^{6,7}$ SCC is the most common histology in Eastern Europe and Asia, and adenocarcinoma is most common in North America and Western Europe. Tobacco and alcohol consumption are major risk factors for esophageal SCC and obesity has been established as the strongest risk factor for esophageal and EGJ adenocarcinoma. ${ }^{1,8-12}$ SCC has become less common in the West over recent decades due to reductions in tobacco and alcohol use, and now accounts for $<30 \%$ of all esophageal cancers in the United States and Western Europe. ${ }^{1}$ In contrast, the incidence of esophageal adenocarcinoma has increased in the West, likely reflecting rising rates of obesity. ${ }^{1}$

In North America, where screening programs for early detection of esophageal and EGJ cancers are not in use or practical because of low incidence, diagnosis is often made late in the disease course. At diagnosis, nearly $50 \%$ of patients have cancer that extends beyond the locoregional confines of the primary tumor and $<60 \%$ of patients with locoregional cancers can undergo a curative resection. Approximately $70 \%$ to $80 \%$ of resected specimens harbor metastases in the regional lymph nodes. Thus, patients in North America often have advanced-stage disease at the time of initial diagnosis, which is reflected by the low survival rates seen with esophageal and EGJ cancers in this region.

This selection from the NCCN Clinical Practice Guidelines in Oncology (NCCN Guidelines) for Esophageal and Esophagogastric Junction Cancers focuses on the 


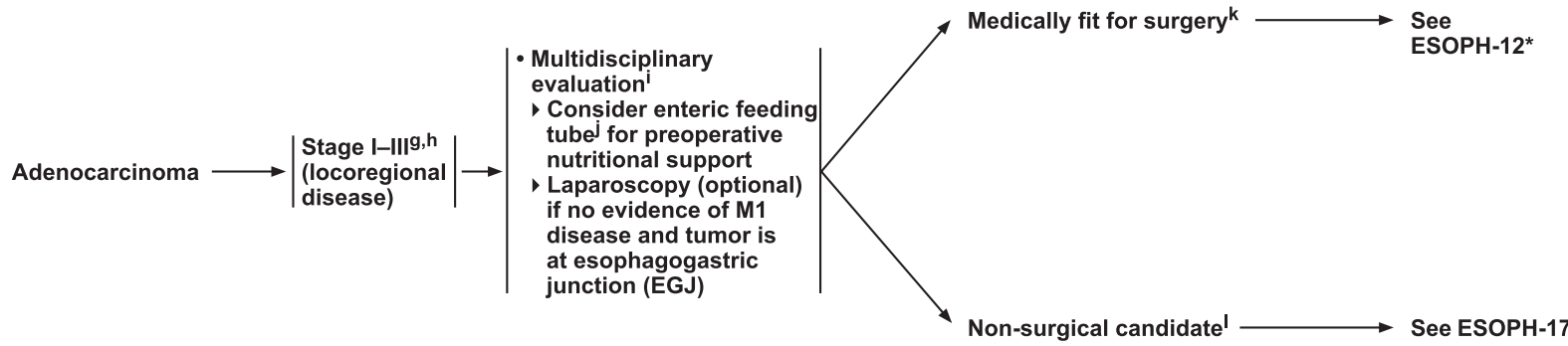

*Available online, in these guidelines, at NCCN.org.

gSee Staging $\left(\mathrm{ST}-1^{*}\right)$ for tumor classification.

hCeliac nodal involvement in cancers of the esophagogastric junction/distal esophagus may still be considered for combined modality therapy.

isee Principles of Multidisciplinary Team Approach for Esophagogastric Cancers (ESOPH-E*).

jPercutaneous gastrostomy tube may be considered for patients with cervical esophageal tumors receiving definitive chemoradiation or for patients with marginally resectable disease. Multidisciplinary expertise is recommended prior to placement of percutaneous gastrostomy tube. The approach, timing, and location of the feeding tube should be discussed with the surgeon prior to its placement.

kMedically able to tolerate major surgery.

IMedically unable to tolerate major surgery or medically fit patients who decline surgery.

management of locally advanced and metastatic adenocarcinoma of the esophagus and EGJ (to view the complete and most recent version of these guidelines, visit NCCN.org).

\section{Staging}

The tumor $(\mathrm{T})$, node $(\mathrm{N})$, and metastasis $(\mathrm{M})$ staging system used by the AJCC is the internationally accepted standard for cancer staging and is a major factor influencing prognosis and treatment decisions. The eighth edition of the AJCC Cancer Staging Manual provides additional resources for esophageal and EGJ cancers not available in the seventh edition, including the incorporation of newly constructed clinical (c) and postneoadjuvant pathologic (yp) stage groupings, to fulfill unmet needs in staging patients under different circumstances. The stage groupings presented in the eighth edition are based on updated data with a significantly increased sample size and number of risk adjustment variables. The current stage groupings were determined using a risk-adjusted random survival forest analysis of collated data generated by the Worldwide Esophageal Cancer Collaboration (WECC) for 22,654 patients spanning 6 continents who were treated with esophagectomy alone or esophagectomy with preoperative and/or postoperative therapy. ${ }^{7}$ Use of these data reflects the current preference for treating locally advanced esophageal cancers with preoperative therapy and represents a major advancement over the seventh edition, which was entirely based on data from patients treated with esophagectomy alone. The availability of these data led to the ability to explicitly define cTNM and ypTNM cohorts and stages. The larger dataset also allowed for better separation of SCC and adenocarcinoma staging. ${ }^{7}$ However, limitations of this dataset still remain, including missing patient variables, heterogeneity of clinical staging among different centers, and poor representation of patients with untreatable or inoperable cancers, such as T4b and M1 cancers. Additionally, the exact modalities used to arrive at the initial clinical stages were not available for analysis. Nevertheless, the eighth edition of the AJCC Cancer Staging Manual represents the best worldwide clinical esophageal cancer staging data currently available.

In esophageal cancer, patient survival is best correlated with pathologic (p) stage, regardless of whether the patient has received preoperative therapy. ${ }^{7}$ Survival analysis of these data revealed that survival decreased with increasing anatomic tumor size and depth (pT), presence of regional lymph node metastases $(\mathrm{pN})$, presence 


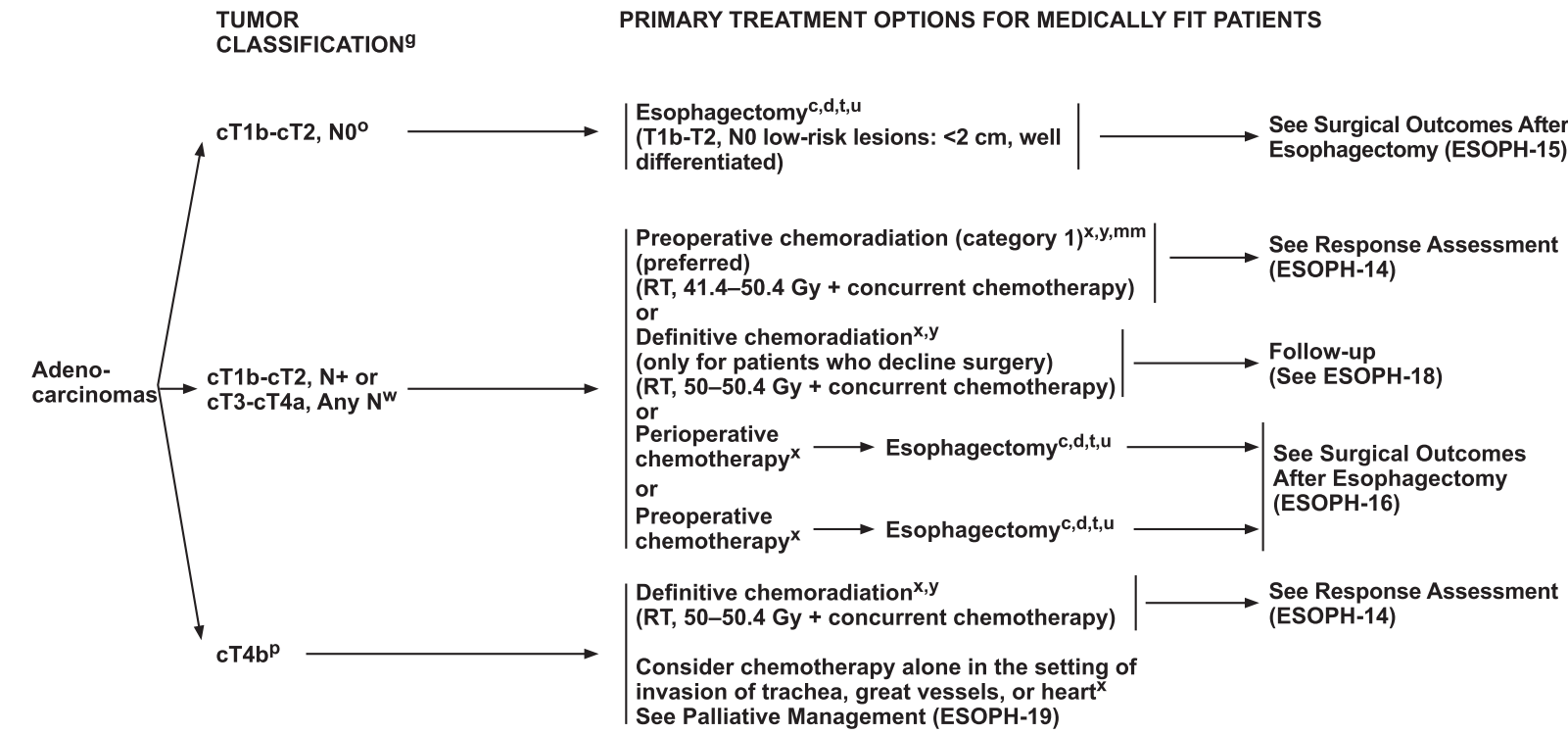

cSee Principles of Pathologic Review and Biomarker Testing (ESOPH-B).

dSee Principles of Surgery (ESOPH-C ${ }^{\star}$ ).

gSee Staging $\left(\mathrm{ST}-1^{*}\right)$ for tumor classification.

oPreclinical staging cannot establish the number of positive nodes.

PFor select patients, consider endoluminal stenting when appropriate.

PFor select patients, consider endoluminal stenting when appropriate
See Principles of Palliative/Best Supportive Care (ESOPH-H ${ }^{*}$ ).

tTranshiatal or transthoracic, or minimally invasive; gastric reconstruction preferred.

uFeeding jejunostomy for postoperative nutritional support, generally preferred.

wHistologic confirmation of suspected positve node is desirable. $\times$ See Principles of Systemic Therapy (ESOPH-F).

y See Principles of Radiation Therapy (ESOPH-G*).

ySee Principles of Radiation Therapy (ESOPH-G*).
mmPreoperative chemoradiation (category 1 ) is preferred over preoperative

chemotherapy for EGJ. (van Hagen P, Hulshof MC, van Lanschot JJ, et al. CROSS

N Engl J Med 2012;366:2074-2084)

*Available online, in these guidelines, at NCCN.org.

of distant metastases (pM), increasing histologic grade (G1-4), and advancing age. ${ }^{13,14}$ Survival increased with a more distal location of cancer within the esophagus. In addition, survival was significantly affected by histopathologic type, with SCC having worse survival than adenocarcinoma. ${ }^{14}$ Analysis of this larger dataset also illuminated significant differences in outcome when comparing the same stage groups between patients receiving preoperative therapy versus those treated with surgery alone. This emphasizes the importance of having separate $\mathrm{p}$ and yp stage groupings to stage patients more accurately within each treatment algorithm. Although surgical pathology yields the most accurate staging, advances in endoscopic techniques and imaging modalities such as endoscopic ultrasound (EUS), CT, and 18-fluorodeoxyglucose (FDG)-PET/CT have greatly improved the accuracy of clinical staging. ${ }^{15}$

\section{Siewert Classification of EGJ Adenocarcinoma}

Siewert classification divides EGJ adenocarcinoma into 3 types based purely on the anatomic location of the epicenter of the tumor or the majority of the tumor mass. ${ }^{16}$ Siewert type I tumors are defined as an adenocarcinoma of the lower esophagus with the tumor epicenter located within 1 to $5 \mathrm{~cm}$ above the anatomic
EGJ. ${ }^{17}$ Siewert type II tumors are defined as a true carcinoma of the cardia with the tumor epicenter located within $1 \mathrm{~cm}$ above and $2 \mathrm{~cm}$ below the EGJ. Siewert type III tumors are defined as a subcardial carcinoma with the tumor epicenter located between 2 to $5 \mathrm{~cm}$ below the EGJ, which infiltrates the EGJ and the lower esophagus from below. In the eighth edition of the AJCC Cancer Staging Manual, EGJ tumors with epicenters located within $2 \mathrm{~cm}$ of the proximal stomach (Siewert types I and II) are staged as esophageal adenocarcinoma. ${ }^{7}$ EGJ tumors with epicenters located $>2 \mathrm{~cm}$ into the stomach (Siewert type III) are now staged using the gastric cancer staging system. In general, Siewert types I and II tumors should be managed in accordance with guidelines for esophageal and EGJ cancers, while Siewert type III tumors are more appropriately managed in accordance with guidelines for gastric cancer.

\section{Principles of Biomarker Testing}

Presently, molecular testing for HER2 status, microsatellite instability status, and programmed deathligand 1 (PD-L1) expression are used in the clinical management of locally advanced, unresectable, and metastatic esophageal and EGJ cancers. 


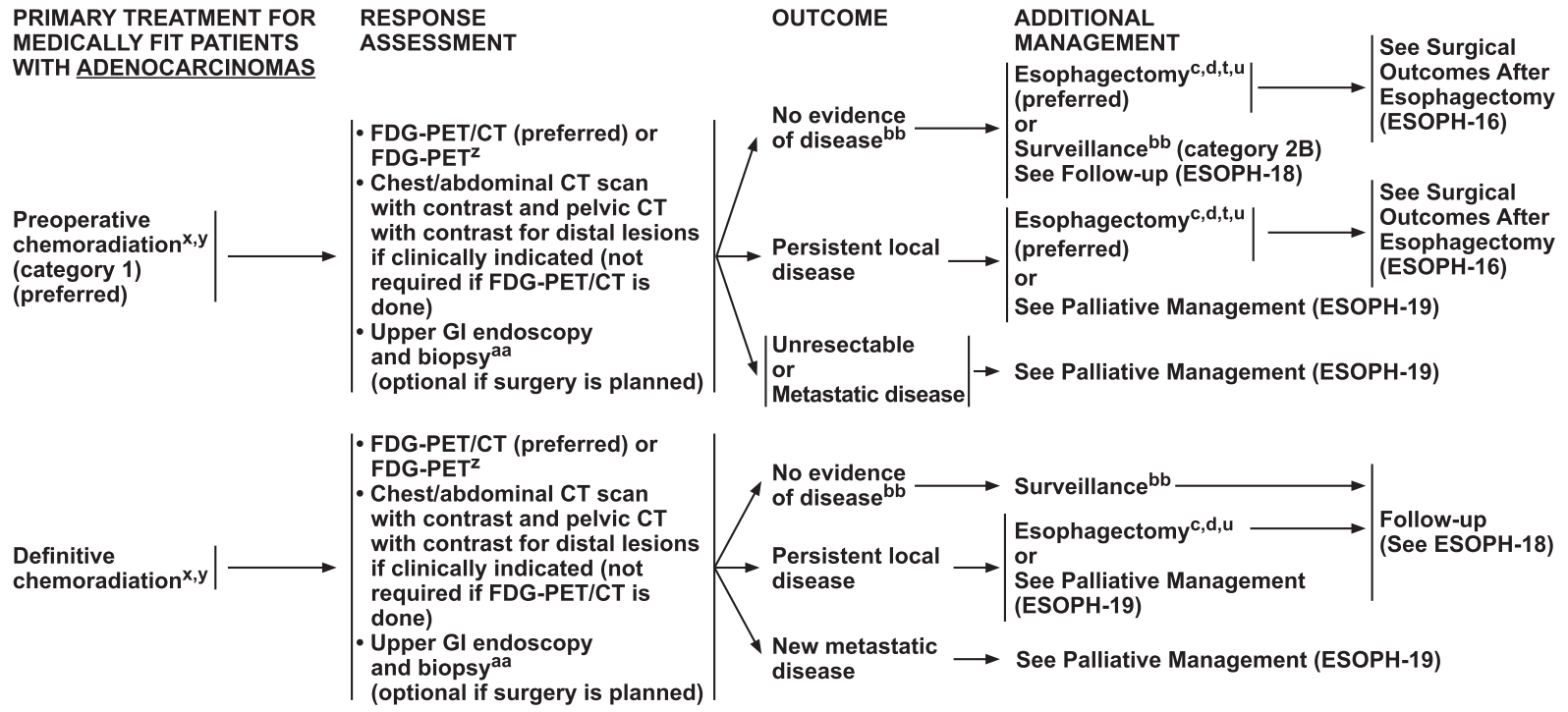

'See Principles of Pathologic Review and Biomarker Testing (ESOPH-B).

dSee Principles of Surgery (ESOPH-C*).

tTranshiatal or transthoracic, or minimally invasive; gastric reconstruction preferred.

uFeeding jejunostomy for postoperative nutritional support, generally preferred.

xSee Principles of Systemic Therapy (ESOPH-F).

ysee Principles of Radiation Therapy (ESOPH-G*).

ySee Principles of Radiation Therapy (ESOPH-G*).
zAssessment $\geq 5-8$ weeks after completion of preoperative therapy.
aaSee Post-Treatment Surveillance-Principles of Endoscopic Staging

aaSee Post-Treatment Surveillance-Principles of Endoscopic Staging and Therapy (ESOPH-A 4 of $5^{*}$ ).

$\mathrm{bb}$ If surveillance is being considered for potentially operable patients, upper GI endoscopy and biopsy should be done.

*Available online, in these guidelines, at NCCN.org.

\section{Assessment of HER2 Positivity}

Overexpression or amplification of the HER2 gene or protein has been implicated in the development of esophageal and EGJ cancers. ${ }^{18}$ However, unlike in breast cancer, the prognostic significance of HER2 status in esophageal and EGJ cancer is unclear. Some studies have reported that HER2 positivity is correlated with tumor invasion and lymph node metastasis, and thus indicates a poor prognosis. ${ }^{19,20}$ Although further studies are needed to assess the prognostic significance of HER2 status in esophageal cancer, the addition of HER2 monoclonal antibodies to chemotherapy regimens is a promising treatment option for patients with HER2positive metastatic disease. The reported rates of HER2 positivity in esophageal and EGJ cancers varies widely $(2 \%-45 \%)^{19}$ and is more frequently seen in adenocarcinoma of the esophagus $(15 \%-30 \%)$ than in SCC $(5 \%-13 \%))^{21-23}$ Additionally, HER2 positivity has been reported to be higher in patients with EGJ adenocarcinomas than in patients with gastric adenocarcinomas. ${ }^{24-26}$ In the ToGA trial that evaluated the addition of trastuzumab to chemotherapy in patients with HER2-positive advanced EGJ or gastric cancers, HER2-positivity rates were 33\% and $21 \%$, respectively, for patients with EGJ and gastric cancers. ${ }^{27}$ Therefore, classification of gastroesophageal cancers based on histologic subtype and primary tumor location may have implications for therapy.

HER2 testing is recommended for all patients with esophageal or EGJ cancer at the time of diagnosis if metastatic disease is documented or suspected (see ESOPH-1, page 856). In concordance with HER2 testing guidelines from the College of American Pathologists (CAP), American Society for Clinical Pathology (ASCP), and $\mathrm{ASCO},{ }^{28}$ the NCCN Guidelines recommend using immunohistochemistry (IHC) and, if needed, in situ hybridization (ISH) techniques to assess HER2 status in esophageal and EGJ cancers (see ESOPH-B page 3 of 5, page 865 ). IHC evaluates the membranous immunostaining of tumor cells, including the intensity and extent of staining and the percentage of immunoreactive tumor cells, with scores ranging from 0 (negative) to $3+$ (positive). In 2008, Hofmann et $\mathrm{al}^{29}$ refined this 4-tiered scoring system to assess HER2 status in gastric cancer by using a cut-off of $\geq 10 \%$ immunoreactive tumor cells for resection specimens. ${ }^{26}$ In a subsequent validation study ( $\mathrm{n}=447$ prospective diagnostic gastric cancer specimens), this scoring system was found to be reproducible between different pathologists. ${ }^{30}$ This modified HER2 scoring system is therefore recommended by the panel. A score of 0 (membranous reactivity in $<10 \%$ of cancer cells) or $1+$ (faint membranous reactivity in $\geq 10 \%$ of cancer cells) 


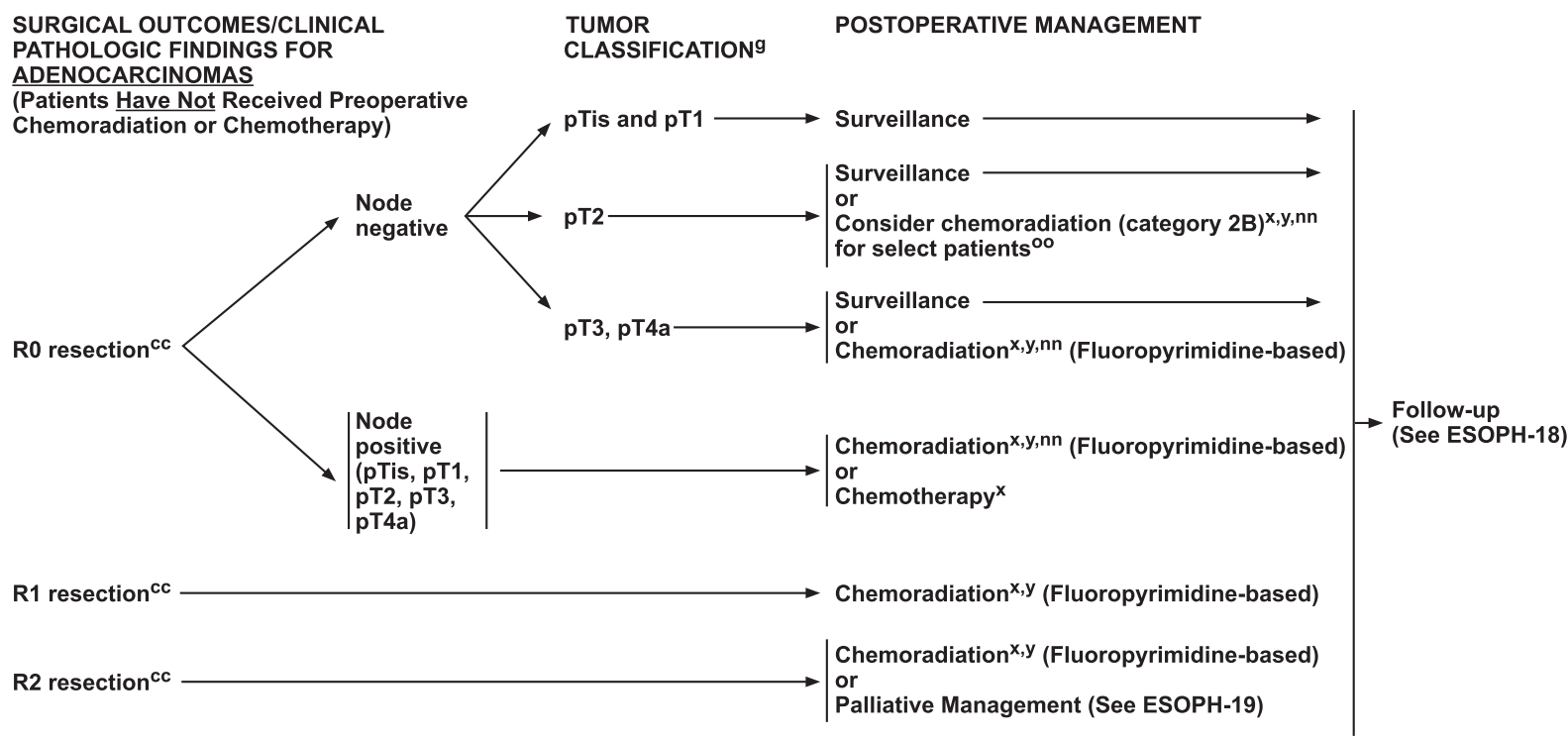

gSee Staging $\left(\mathrm{ST}-1^{*}\right)$ for tumor classification.

xSee Principles of Systemic Therapy (ESOPH-F)

y

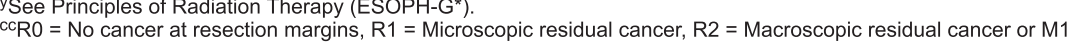

${ }^{n n}$ Smalley SR, Benedetti JK, Haller DG, et al. Updated analysis of SWOG-directed intergroup study 0116: a phase III trial of adjuvant radiochemotherapy versus

observation after curative gastric cancer resection. J Clin Oncol 2012;30:2327-2333. See Principles of Systemic Therapy (ESOPH-F).

${ }^{\circ} \mathrm{C}$ Consider chemoradiation for patients with high-risk lower esophagus or EGJ adenocarcinoma. High-risk features include poorly differentiated or higher grade cancer,

lymphovascular invasion, perineural invasion, or $<50$ years of age.

*Available online, in these guidelines, at NCCN.org.

is considered to be HER2-negative. A score of $2+$ (weak to moderate membranous reactivity in $\geq 10 \%$ of cancer cells) is considered equivocal and should be additionally examined by fluorescence in situ hybridization (FISH) or other ISH methods. FISH/ISH results are expressed as the ratio between the number of copies of the HER2 gene and the number of chromosome 17 centromeres (CEP17) within the nucleus counted in at least 20 cancer cells (HER2:CEP17). Alternatively, FISH/ISH results may be given as the average HER2 copy number per cell. Cases that have an IHC score of $3+$ (strong membranous reactivity in $\geq 10 \%$ of cancer cells) or an IHC score of $2+$ and are FISH/ISH positive (HER2:CEP17 ratio $\geq 2$ or average HER 2 copy number $\geq 6$ signals/cell) are considered HER2 positive. Positive $(3+)$ or negative $(0$ or 1+) HER2 IHC results do not require further ISH testing.

\section{Assessment of Microsatellite Instability and}

\section{PD-L1 Expression}

In its first-ever site-agnostic approval, the U.S. FDA approved pembrolizumab for the treatment of unresectable or metastatic microsatellite instability-high (MSI-H) or deficient mismatch repair (dMMR) solid tumors in the second-line or subsequent setting. ${ }^{31}$
Therefore, MSI-H/dMMR status should be assessed in all patients with esophageal or EGJ adenocarcinoma if metastatic disease is documented or suspected (see ESOPH-1, page 856). MMR status is assessed by IHC staining to measure expression levels of proteins involved in DNA mismatch repair (ie, MLH1, MSH2, MSH6, PMS2).$^{32}$ MSI is assessed by polymerase chain reaction to measure gene expression levels of microsatellite markers (ie, BAT25, BAT26, MONO27, NR21, NR24). ${ }^{33}$ It should be noted that IHC for MMR and polymerase chain reaction for MSI are different assays measuring the same biologic effect. Testing is performed on formalin-fixed, paraffinembedded tissue and results are interpreted as MSI-H or dMMR in accordance with CAP DNA Mismatch Repair Biomarker Guidelines. ${ }^{34}$

In addition, pembrolizumab has been granted accelerated FDA approval as a third- or subsequent-line treatment option for patients with recurrent locally advanced or metastatic EGJ adenocarcinoma whose tumors express PD-L1 with a combined positive score (CPS) $\geq 1$ as determined by an FDA-approved companion diagnostic test (see ESOPH-B page 4 of 5, page 866). ${ }^{35,36}$ This is a qualitative IHC assay using anti-PD-L1 antibodies for the detection of PD-L1 protein levels in formalin-fixed, paraffin-embedded tumor tissue. CPS is determined by 


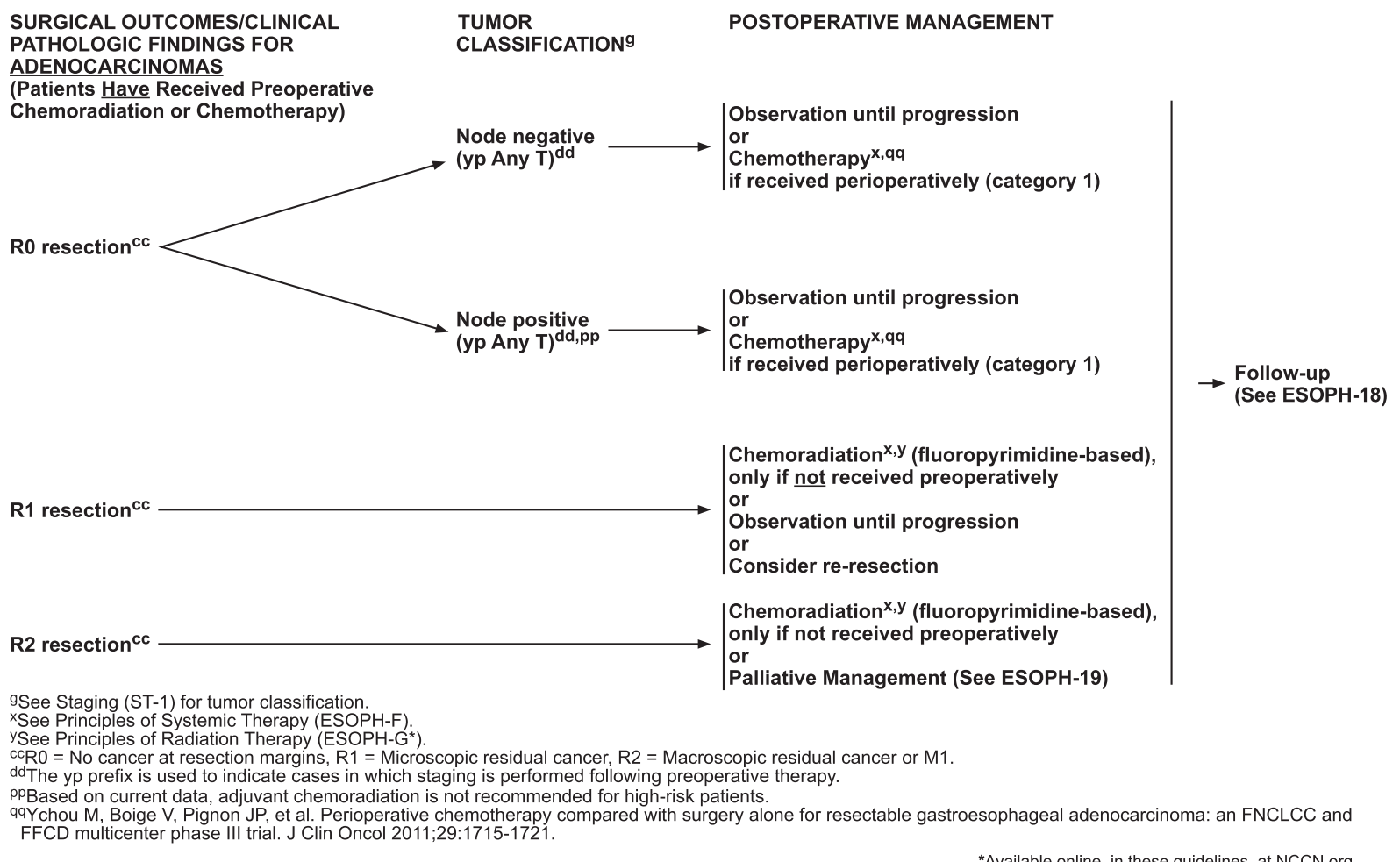

*Available online, in these guidelines, at NCCN.org.

the number of PD-L1 staining cells (tumor cells, lymphocytes, macrophages) divided by the total number of viable tumor cells evaluated, multiplied by 100 . The panel recommends that this pembrolizumab treatment option also be extended to patients with esophageal adenocarcinomas with PD-L1 expression levels by CPS of $\geq 1$. The panel also recommends second-line treatment with pembrolizumab for esophageal cancers with PD-L1 expression levels by CPS of $\geq 10$ (category 2B). ${ }^{37}$ Therefore, PD-L1 testing is recommended for all patients with esophageal or EGJ cancers if metastatic disease is documented or suspected.

\section{Principles of Surgery}

Surgery is a major component of treatment of locoregional esophageal and EGJ cancers. Improvements in staging techniques, patient selection, postsurgical care, and surgical experience have led to a marked reduction in surgical morbidity and mortality in recent years. Additionally, randomized trials have shown that preoperative chemoradiation ${ }^{38}$ and perioperative chemotherapy ${ }^{39,40}$ have significantly improved survival in patients with resectable, locoregionally advanced esophageal and EGJ cancers.
All patients should be evaluated to determine whether they are medically fit enough to tolerate general anesthesia and major abdominal and/or thoracic surgery. ${ }^{41}$ Before surgery, clinical staging should be performed to assess resectability with CT scan of the chest and abdomen, whole-body FDG-PET (integrated FDG-PET/CT scan is preferred), and EUS. ${ }^{42}$ Esophagectomy should be considered for all medically fit patients with resectable esophageal cancer ( $>5 \mathrm{~cm}$ from cricopharyngeus) Cervical or cervicothoracic esophageal cancers $<5 \mathrm{~cm}$ from the cricopharyngeus should be treated with definitive chemoradiation. Enteral nutritional support should be considered for patients with significant dysphagia and/or weight loss before or during induction therapy. A jejunostomy feeding tube is preferred over a gastrostomy feeding tube for preoperative nutritional support because placement of a gastrostomy tube may compromise the integrity of gastric conduit for reconstruction.

The Siewert tumor type should be assessed in all patients with adenocarcinomas involving the EGJ. The surgical approaches for Siewert type I and II tumors are similar to those described previously. Siewert type III tumors are considered gastric cancers and the surgical approach for these tumors is described in the NCCN Guidelines for Gastric Cancer (available at NCCN.org). ${ }^{16,43,44}$ 


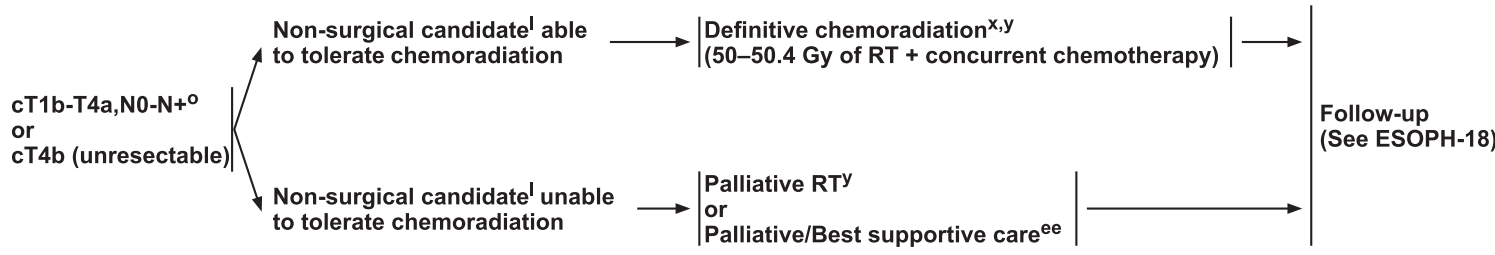

\footnotetext{
aSee Principles of Endoscopic Staging and Therapy (ESOPH-A*).

gSee Staging $\left(\mathrm{ST}-1^{*}\right)$ for tumor classification.

IMedically unable to tolerate major surgery or medically fit patients who decline surgery.

mpTis, pT1a, and pT1b tumor classification are defined by pathology of the diagnostic

ER specimen See Principles of Endoscopic Staging and Therapy (ESOPH-A*).

${ }^{n}$ The initial diagnostic ER procedure may prove therapeutic for some patients, but for

others additional therapy may be necessary prior to the start of surveillance.

opreclinical staging cannot establish the number of positive nodes.

x See Principles of Systemic Therapy (ESOPH-F).

ySee Principles of Radiation Therapy (ESOPH-G*).

eesee Principles of Palliative/Best Supportive Care (ESOPH-H*).

ffoor prognostic features include lymphovacular invasion (LVI), poorly

differentiated histology, positive margin(s), and/or maximum tumor diameter $2 \mathrm{~cm}$

or more.

kkER followed by ablation may be used to completely eliminate residual dysplasia

or Barrett's epithelium.
}

In some cases, additional esophageal resection may be necessary to obtain adequate surgical margins. Laparoscopy may be useful in select patients for the detection of radiographically occult metastatic disease, especially in patients with Siewert type II and III tumors. ${ }^{45}$ Positive peritoneal cytology in the absence of visible peritoneal metastases is associated with poor prognosis in patients with EGJ adenocarcinoma. ${ }^{46}$ Patients with advanced tumors or node-positive tumors should be considered for laparoscopic staging with peritoneal washings.

Lymph node dissection (lymphadenectomy) can be performed using the standard or extended (en-bloc) technique. The number of lymph nodes removed has been shown to be an independent predictor of survival after esophagectomy. ${ }^{47,48}$ In a retrospective analysis of 4,882 patients in the SEER database, patients diagnosed with invasive esophageal cancer who had $\geq 12$ lymph nodes examined had significantly reduced mortality compared with those who had 0 to 11 lymph nodes examined; patients who had $\geq 30$ lymph nodes examined had the lowest mortality of any group. ${ }^{49}$ A report from the WECC database, which analyzed 4,627 patients who had esophagectomy without preoperative therapy, suggested that a greater extent of lymphadenectomy was associated with increased survival for all patients with node-positive cancers. ${ }^{48}$ Based on this study, optimum lymphadenectomy in node-positive cancers was 10 nodes for pT1, 15 nodes for pT2, and 29 to 50 nodes for pT3/T4. Therefore, the NCCN Guidelines recommend that a thorough dissection be performed to identify all lymph nodes with at least 15 lymph nodes submitted for pathologic evaluation and adequate nodal staging in patients undergoing esophagectomy without preoperative chemoradiation.

\section{Combined Modality Therapy}

Combined modality therapy has been shown to significantly increase survival in patients with esophageal or EGJ cancer with locoregional disease compared with resection alone. ${ }^{50-52}$ Preoperative chemoradiation is the preferred approach for localized resectable disease. ${ }^{38}$ Perioperative chemotherapy or preoperative chemotherapy are alternative options for adenocarcinoma of the thoracic esophagus or EGJ. ${ }^{39,40,53}$ Other treatment options include postoperative chemoradiation ${ }^{54,55}$ and postoperative chemotherapy. ${ }^{56}$ Definitive chemoradiation should be reserved for patients with unresectable disease or those who decline surgery. ${ }^{57-60}$ See ESOPH-F page 2 of 13 (page 868) for recommended regimens for combined modality therapy. 


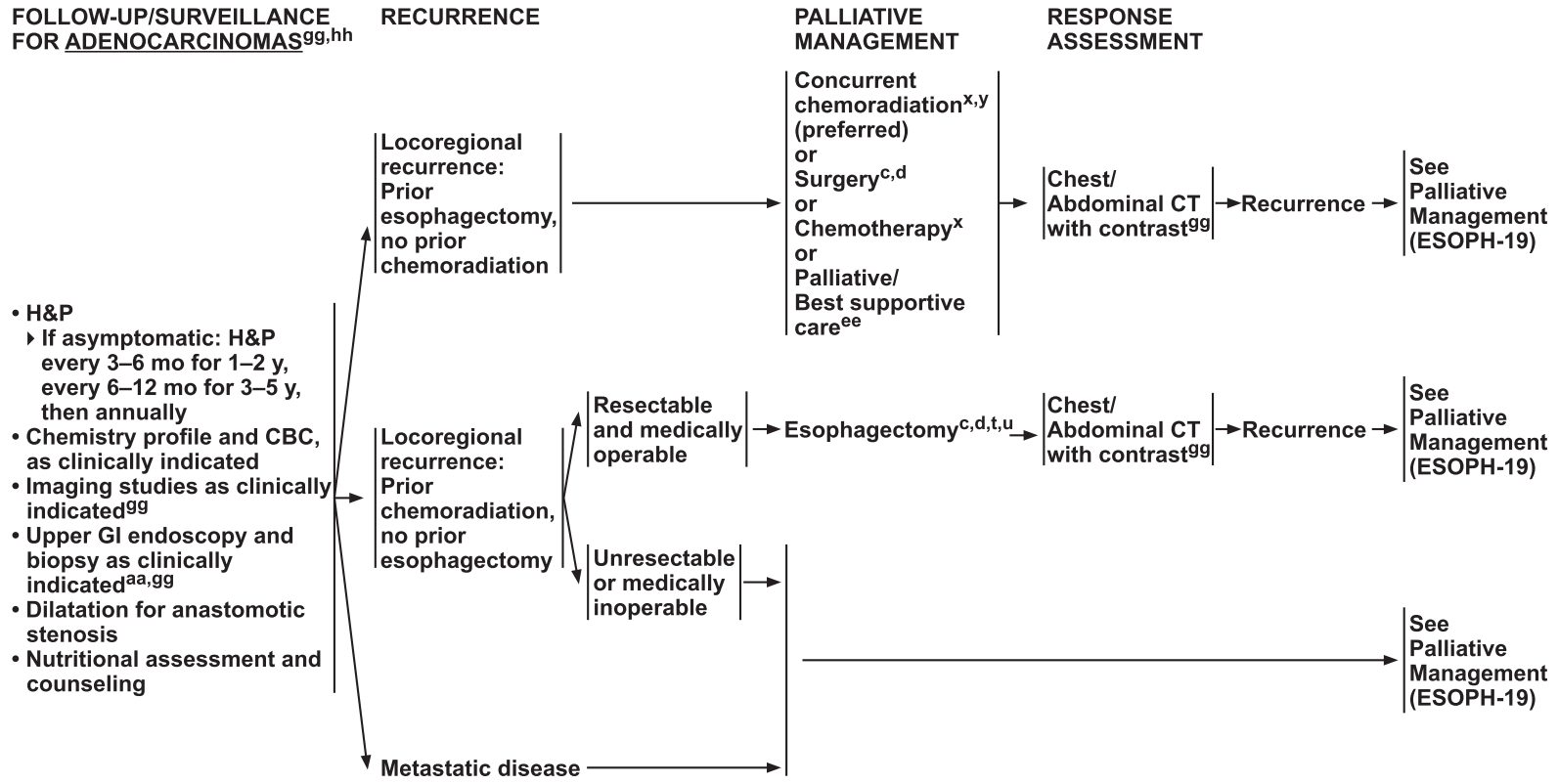

'See Principles of Pathologic Review and Biomarker Testing (ESOPH-B).

dSee Principles of Surgery (ESOPH-C*).

dSee Principles of Surgery (ESOPH-C*).
tTranshiatal or transthoracic, or minimally invasive; gastric reconstruction preferred.

uFeeding jejunostomy for postoperative nutritional support, generally preferred.

xSee Principles of Systemic Therapy (ESOPH-F)

ySee Principles of Radiation Therapy $\left(\mathrm{ESOPH}-\mathrm{G}^{*}\right)$.

aasee Post-Treatment Surveillance-Principles of Endoscopic Staging and Therapy

(ESOPH-A 4 of $5^{*}$ ).

eesee Principles of Palliative/Best Supportive Care $\left(\mathrm{ESOPH}-\mathrm{H}^{*}\right)$.

ggSee Principles of Surveillance (ESOPH-I*).

hh See Principles of Survivorship (ESOPH-J*).

*Available online, in these guidelines, at NCCN.org.

\section{Preoperative Chemoradiation Therapy}

Preoperative chemoradiation is associated with improved overall survival (OS), disease-free survival (DFS), and pathologic complete response (pCR) compared with preoperative chemotherapy or surgery alone in patients with locoregional esophageal cancer. ${ }^{61-67}$ Results from the multicenter phase III randomized CROSS trial, the largest trial in its class, showed that preoperative chemoradiation with paclitaxel and carboplatin significantly improved OS and DFS compared with surgery alone in patients with resectable esophageal or EGJ cancers ( $\mathrm{n}=366$; $75 \%$ had adenocarcinoma). ${ }^{38}$ Median OS was 49 months in the preoperative chemoradiation arm $(n=178)$ compared with 24 months in the surgery alone arm $(n=188$; hazard ratio $[\mathrm{HR}]=0.657 ; 95 \% \mathrm{CI}$, $0.495-0.871 ; P=.003)$. The R0 resection rate was also higher in the preoperative chemoradiation arm compared with the surgery alone arm ( $92 \%$ vs $69 \% ; P<.001)$. The $1-$, $2-, 3-$, and 5-year OS rates were $82 \%, 67 \%, 58 \%$, and $47 \%$, respectively, in the preoperative chemoradiation arm compared with $70 \%, 50 \%, 44 \%$, and $34 \%$, respectively, in the surgery alone arm.

After a minimum follow-up of 24 months, the overall rate of recurrence was $35 \%$ in the preoperative chemoradiation arm compared with $58 \%$ in the surgery alone arm. ${ }^{68}$ Additionally, preoperative chemoradiation significantly reduced locoregional recurrence from $34 \%$ to $14 \%$ $(P<.001)$ and peritoneal carcinomatosis from $14 \%$ to $4 \%$ $(P<.001){ }^{68}$ Importantly, preoperative chemoradiation did not negatively impact postoperative health-related quality of life compared with surgery alone in patients participating in the CROSS trial. ${ }^{69} \mathrm{~A}$ study reporting the long-term results of the CROSS trial verified that median OS was significantly improved in the preoperative chemoradiation group. ${ }^{70}$ After a median followup of 84.1 months, median OS was 48.6 months in the preoperative chemoradiation group compared with 24 months in the surgery alone group ( $\mathrm{HR}=0.68 ; 95 \%$ CI, 0.53-0.88; $P=.003$ ). Median OS for patients with adenocarcinoma was 43.2 months and 27.1 months, respectively $(P=.038)$. These results confirmed the survival benefit for preoperative chemoradiation therapy with paclitaxel and carboplatin in patients with resectable esophageal or EGJ cancers. Therefore, the panel recommends combined paclitaxel and carboplatin as a category 1 preferred regimen for preoperative chemoradiation.

The panel also recommends FOLFOX (fluorouracil/ leucovorin calcium/oxaliplatin) as a category 1 preferred option for preoperative chemoradiation. The efficacy 


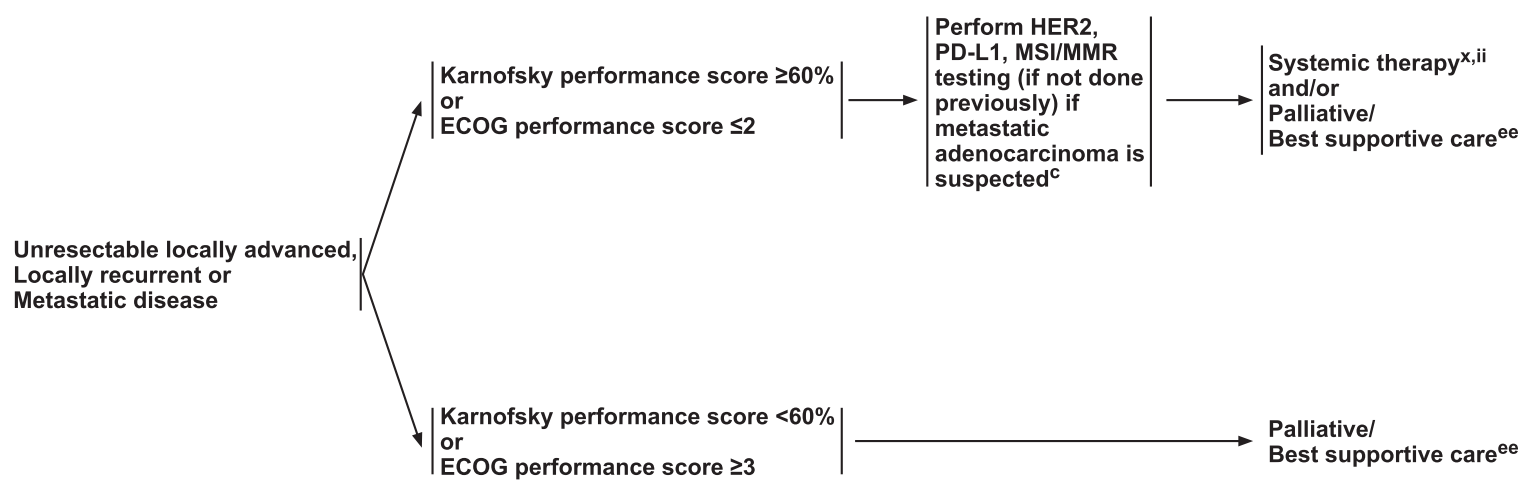

*Available online, in these guidelines, at NCCN.org.

'See Principles of Pathologic Review and Biomarker Testing (ESOPH-B).

'See Principles of Systemic Therapy (ESOPH-F)

eesee Principles of Palliative/Best Supportive Care (ESOPH-H*).

iiFurther treatment after two sequential regimens should be dependent upon performance status and availability of clinical trials

and safety of preoperative FOLFOX combined with radiation therapy (RT) was evaluated in a single-arm phase II SWOG trial involving 93 patients with clinically staged II or III esophageal adenocarcinoma. ${ }^{71}$ Twenty-six patients (28\%) had confirmed pCR (95\% CI, 19.1\%-38.2\%) and $19.4 \%$ of patients experienced grade 4 treatmentrelated toxicities. At a median follow-up of 39.2 months, estimates of median and 3-year OS were 28.3 months and $45.1 \%$, respectively. A small trial of 38 patients with stage II-IV esophageal adenocarcinoma also showed that FOLFOX combined with RT is safe and effective in the preoperative setting, with $38 \%$ of patients attaining pCR. ${ }^{72}$ Other recommended regimens for preoperative chemoradiation include fluorouracil and cisplatin (category 1), ${ }^{73,74}$ irinotecan and cisplatin (category 2B), ${ }^{75}$ and paclitaxel and a fluoropyrimidine (fluorouracil or capecitabine [category 2B]). ${ }^{76}$ CALGB 9781 was a prospective phase III trial that randomized patients $(n=56)$ with stage I-III esophageal cancers to receive preoperative chemoradiation with fluorouracil and cisplatin followed by surgery $(n=30)$ or surgery alone $(n=26) .^{73}$ After a median follow-up of 6 years, the median OS was 4.5 years in the preoperative chemoradiation group versus 1.8 years in the surgery alone group $(P=.002)$. Patients receiving preoperative chemoradiation also had an improved 5-year OS rate (39\% vs $16 \%$ ). The results from this trial reflect a long-term survival advantage with the use of preoperative chemoradiotherapy with fluorouracil and cisplatin in the treatment of esophageal cancer. Irinotecan and cisplatin showed modest activity in a single-institution retrospective trial involving patients $(n=44)$ with locally advanced esophageal carcinoma. ${ }^{75}$ All patients underwent $\mathrm{R} 0$ resection, and the pCR rate was $25 \%$. The median DFS and OS were 24 months and 34 months, respectively, and the 3 -year OS rate was $46 \%$.

\section{Perioperative Chemotherapy}

The survival benefit of perioperative chemotherapy in gastroesophageal cancers was first demonstrated in the landmark phase III MAGIC trial. ${ }^{77}$ This study, which compared perioperative chemotherapy with epirubicin, cisplatin, and fluorouracil (ECF) to surgery alone, established that perioperative chemotherapy improves progression-free survival (PFS) and OS in patients with nonmetastatic stage II and higher gastric or EGJ adenocarcinoma. In the randomized controlled phase II/III FLOT4 trial, Al-Batran et $\mathrm{al}^{40}$ compared perioperative chemotherapy with fluorouracil, leucovorin, oxaliplatin, and docetaxel (FLOT) to the standard ECF regimen in patients 


\section{Assessment of Overexpression or Amplification of HER2 in Esophageal and Esophagogastric Junction Cancers}

For patients with inoperable locally advanced, recurrent, or metastatic adenocarcinoma of the esophagus or EGJ for whom trastuzumab therapy is being considered, assessment for tumor HER2 overexpression using immunohistochemistry (IHC) and fluorescence in situ hybridization (FISH) or other in situ hybridization methods is recommended. ${ }^{11}$

\begin{tabular}{|c|l|l|l|}
\hline \multicolumn{1}{|l|}{ Iable 3} & \begin{tabular}{l} 
Immunohistochemical Criteria for Scoring HER2 Expression in Esophageal and Esophagogastric Junction Cancers, \\
\hline Immunohistochemistry
\end{tabular} & $\begin{array}{l}\text { HER2 Overexpression } \\
\text { Assessment }\end{array}$ \\
\hline 0 & $\begin{array}{l}\text { No reactivity or membranous } \\
\text { reactivity in <10\% of cancer cells }\end{array}$ & $\begin{array}{l}\text { No reactivity or no membranous reactivity in any } \\
\text { cancer cell }\end{array}$ & Negative \\
\hline $1+$ & $\begin{array}{l}\text { Faint or barely perceptible membranous } \\
\text { reactivity in } \geq 10 \% \text { of cancer cells; cells are } \\
\text { reactive only in part of their membrane }\end{array}$ & $\begin{array}{l}\text { Cluster of five or more cancer cells with a faint or barely } \\
\text { perceptible membranous reactivity irrespective of } \\
\text { percentage of cancer cells positive }\end{array}$ & Negative \\
\hline $2+$ & $\begin{array}{l}\text { Weak to moderate complete, basolateral } \\
\text { or lateral membranous reactivity in } \geq 10 \% \\
\text { of cancer cells }\end{array}$ & $\begin{array}{l}\text { Cluster of five or more cancer cells with a weak to } \\
\text { moderate complete, basolateral, or lateral membranous } \\
\text { reactivity irrespective of percentage of cancer cells } \\
\text { positive }\end{array}$ & Equivocal \\
\hline $3+$ & $\begin{array}{l}\text { Strong complete, basolateral, or lateral } \\
\text { membranous reactivity in } \geq 10 \% \text { of } \\
\text { cancer cells }\end{array}$ & $\begin{array}{l}\text { Cluster of five or more cancer cells with a strong } \\
\text { complete, basolateral, or lateral membranous reactivity } \\
\text { irrespective of percentage of cancer cells positive }\end{array}$ \\
\hline
\end{tabular}

'The NCCN Guidelines Panel recommends that HER2 immunohistochemistry be ordered/performed first, followed by in situ hybridization (ISH) methods in cases showing 2+ (equivocal) expression by IHC. Positive (3+) or negative (0 or 1+) HER2 IHC results do not require further ISH testing. Cases with HER2:CEP17 ratio $\geq 2$ or an average HER2 copy number $\geq 6.0$ signals/cell are considered positive by ISH/FISH.

jReprinted and adapted from Bartley AN, Washington MK, Colasacco C, et al. HER2 testing and clinical decision making in gastroesophageal adenocarcinoma: guidelin from the College of American Pathologists, American Society for Clinical Pathology, and the American Society of Clinical Oncology. J Clin Oncol 2017;35:446-464 with permission from the American Society of Clinical Oncology.

1 Bartley AN, Washington MK, Colasacco C, et al. HER2 testing and clinical decision making in gastroesophageal adenocarcinoma: guideline from the College of American Pathologists, American Society of Clinical Pathology, and American Society of Clinical Oncology. J Clin Oncol 2017 Feb;35:446-464.

with resectable nonmetastatic gastric or EGJ adenocarcinoma. In the phase II part of the study, 265 patients were randomized to receive either 3 preoperative and postoperative cycles of ECF $(n=137)$ or 4 preoperative and postoperative cycles of FLOT $(n=128)$. Results showed that FLOT was associated with significantly higher proportions of patients attaining pCR than was ECF (16\%; 95\% CI, 10\%-23\% vs $6 \%$; 95\% CI, $3-11 ; P=.02) .{ }^{40}$ Additionally, FLOT was associated with a reduction in the percentage of patients experiencing at least one grade 3-4 adverse event, including neutropenia, leucopenia, nausea, infection, fatigue, and vomiting ( $40 \%$ of patients in the ECF group vs $25 \%$ of patients in the FLOT group). In the phase III part of the trial, 716 patients were randomized to receive FLOT $(n=356)$ or ECF $(n=360) .{ }^{78}$ Results showed that median OS was increased in the FLOT group compared with the ECF group (50 vs 35 months; $\mathrm{HR}=0.77$; 95\% CI, 0.63-0.94). The percentage of patients with serious chemotherapyrelated adverse events was the same in the 2 groups ( $27 \%$ in the ECF group vs $27 \%$ in the FLOT group). Therefore, ECF should no longer be recommended in this setting. However, because of considerable toxicity associated with the FLOT regimen, the panel recommends its use in select patients with good performance status. The preferred perioperative regimen for most patients who have good to moderate performance status is FOLFOX

In the FNCLCC ACCORD 07 trial $(n=224$ patients; $75 \%$ had adenocarcinoma of the lower esophagus or EGJ), Ychou et $\mathrm{al}^{39}$ reported that perioperative chemotherapy with fluorouracil and cisplatin significantly increased the curative resection rate, DFS, and OS in patients with resectable cancer. At a median follow-up of 5.7 years, the 5 -year OS rate was $38 \%$ for patients in the perioperative chemotherapy group and $24 \%$ for patients in the surgery alone group $(P=.02)$. The corresponding 5 -year DFS rates were $34 \%$ and $19 \%$, respectively. Although this trial was prematurely terminated due to low accrual, the panel believes that perioperative fluorouracil and cisplatin is a viable treatment option for patients with locally advanced resectable esophageal or EGJ cancers.

\section{Postoperative Chemoradiation Therapy}

The landmark Intergroup-0116 (INT-0116) trial investigated the effectiveness of surgery followed by postoperative chemotherapy plus chemoradiation on the survival of patients with resectable adenocarcinoma of the stomach or EGJ. ${ }^{54,55}$ In this trial, 556 patients (stage IB to IV, M0) were randomized to receive surgery followed 
Microsatellite Instability $(\mathrm{MSI})^{\mathrm{k}}$ or Mismatch Repair (MMR) ${ }^{\mathrm{k}}$ Testing

- MMR or MSI testing should be considered on locally advanced, recurrent, or metastatic esophageal and EGJ cancers, ${ }^{12}$ in patients who are candidates for treatment with PD-1 inhibitors. The testing is performed on formalin-fixed paraffin-embedded (FFPE) tissue and results are interpreted as MSI-high or mismatch protein repair-deficient in accordance with CAP DNA Mismatch Repair Biomarker Reporting Guidelines. ${ }^{13}$ MMR or MSI testing should be performed only in CLIA-approved laboratories.

PD-L1 Testing

- PD-L1 testing may be considered on locally advanced, recurrent, or metastatic esophageal and EGJ cancers in patients who are candidates for treatment with PD-1 inhibitors. An FDA-approved companion diagnostic test for use on FFPE tissue is available as an aid in identifying patients for treatment with PD-1 inhibitors. PD-L1 testing should be performed only in CLIA-approved laboratories.

- Assessment of PD-L1 Protein Expression in Esophageal and EGJ Cancers

- This is a qualitative immunohistochemical assay using anti-PD-L1 antibodies for the detection of PD-L1 protein in FFPE tissues from esophageal or EGJ cancers. A minimum of 100 tumor cells must be present in the PD-L1-stained slide for the specimen to be considered adequate for PD-L1 evaluation. A specimen is considered to have PD-L1 expression if the combined positive score (CPS) $\geq 1$. CPS is the number of PD-L1 staining cells (ie, tumor cells, lymphocytes, macrophages) divided by the total number of viable tumor cells, multiplied by 100.

$\mathrm{k}_{\mathrm{IHC}}$ for MMR and PCR for MSI are different assays measuring the same biological effect.

12Le DT, Uram JN, Wang H, et al. PD-1 blockade in tumors with mismatch-repair deficiency. N Engl J Med 2015;372:2509-2520.

${ }^{13}$ Bartley AN, Fitzgibbons PL, Broaddus RR, Shi C. Template for Reporting Results of DNA Mismatch Repair Testing in Patients Being

Considered for Checkpoint Inhibitor Immunotherapy. College of American Pathologists. 2018.

by postoperative chemotherapy plus chemoradiation ( $n=281$; bolus fluorouracil plus leucovorin before and after concurrent chemoradiation with the same regimen) or surgery alone $(n=275) .{ }^{55}$ Most patients had T3 or T4 tumors (69\%) and node-positive disease $(85 \%)$. After a median follow-up of 5 years, median OS in the surgery-only group was 27 months compared with 36 months in the postoperative chemotherapy plus chemoradiation group $(P=.005)$. The postoperative chemotherapy plus chemoradiation group also had better 3-year OS (50\% vs $41 \%$ ) and recurrence-free survival rates (48\% vs $31 \%$ ) than the surgery-only group. There was also a decrease in local failure as the first site of failure in the chemoradiation group ( $19 \%$ vs $29 \%$ ). After a median follow-up of $>10$ years, survival remained improved in patients treated with postoperative chemoradiation. ${ }^{54}$ Additionally, data from a retrospective analysis showed that postoperative chemoradiation according to the INT0116 protocol resulted in improved 3-year DFS rates after curative resection in patients $(n=211)$ with EGJ adenocarcinoma and positive lymph nodes who did not receive neoadjuvant chemotherapy (37\% vs $24 \%$ after surgery alone). ${ }^{79}$

The results of the INT-0116 trial established the efficacy of postoperative chemoradiation in patients with resected gastric or EGJ adenocarcinoma who have not received preoperative therapy. However, the dosing schedule of chemotherapy agents used in this trial was associated with high rates of grade 3-4 hematologic and GI toxicities (54\% and $33 \%$, respectively). Among the 281 patients assigned to the chemoradiation group, $17 \%$ discontinued treatment and 3 patients died as a result of chemoradiation-related toxicities, including pulmonary fibrosis, cardiac event, and myelosuppression. Therefore, the doses and schedule of chemotherapy agents used in the INT-0116 trial are no longer recommended due to concerns regarding toxicity. See "Principles of Systemic Therapy-Regimens and Dosing Schedules (ESOPH-F page 8 of 13, available online, in these guidelines, at NCCN.org) for recommended modifications to this regimen.

\section{Preoperative Chemotherapy}

Clinical trials have investigated chemotherapy alone in the preoperative setting for locally advanced esophageal cancer. ${ }^{53,80-82}$ In the Medical Research Council OEO2 trial, 802 patients with potentially resectable esophageal cancer were randomly assigned to receive either 2 cycles of preoperative fluorouracil and cisplatin followed by surgery or surgery alone. ${ }^{80}$ Median survival was 16.8 months 
- Systemic therapy regimens recommended for advanced esophageal and EGJ adenocarcinoma, SCC of the esophagus, and gastric adenocarcinoma may be used interchangeably (except as indicated)

- Regimens should be chosen in the context of performance status (PS), comorbidities, and toxicity profile.

- Trastuzumab should be added to chemotherapy for HER2 overexpressing metastatic adenocarcinoma.

- Two-drug cytotoxic regimens are preferred for patients with advanced disease because of lower toxicity. Three-drug cytotoxic regimens

should be reserved for medically fit patients with good PS and access to frequent toxicity evaluation.

- Modifications of category 1 regimen or use of category 2A or 2B regimens may be preferred (as indicated), with evidence supporting more

favorable toxicity profile without compromising efficacy. ${ }^{1}$

- Doses and schedules for any regimen that is not derived from category 1 evidence is a suggestion, and subject to appropriate modifications

depending on the circumstances.

- Alternate combinations and schedules of cytotoxics based on the availability of the agents, practice preferences, and contraindications are permitted.

- Preoperative chemoradiation is the preferred approach for localized adenocarcinoma of the thoracic esophagus or EGJ. ${ }^{2}$ Perioperative

chemotherapy is an alternative option for distal esophagus and EGJ.3,4

- In the adjuvant setting, upon completion of chemotherapy or chemoradiation, patients should be monitored for any long-term treatmentrelated complications.

\footnotetext{
1 Van Cutsem E, Moiseyenko VM, Tjulandin S, et al. Phase III study of docetaxel and cisplatin plus fluorouracil compared with cisplatin and fluorouracil as first-line therapy for advanced gastric cancer: a report of the V325 Study Group. J Clin Oncol 2006;24:4991-4997.

2van Hagen P, Hulshof MC, van Lanschot JJ, et al. Preoperative chemoradiotherapy for esophageal or junctional cancer. N Engl J Med 2012;366:2074-2084.

2van Hagen P, Hulshof MC, van Lanschot JJ, et al. Preoperative chemoradiotherapy for esophageal or junctional cancer. N Engl J Med 2012;366:2074-2084. FFCD multicenter phase III trial. J Clin Oncol 2011;29:1715-1721.

${ }^{4}$ Al-Batran S-E, Homann N, Pauligk C, et al. Perioperative chemotherapy with fluorouracil plus leucovorin, oxaliplatin, and docetaxel versus fluorouracil or capecitabine plus

cisplatin and epirubicin for locally advanced, resectable gastric or gastro-oesophageal junction adenocarcinoma (FLOT4): a randomised, phase 2/3 trial. Lancet 2019.
}

in the preoperative chemotherapy group compared with 13.3 months in the surgery alone group, and 2-year survival rates were $43 \%$ and $34 \%$, respectively. Long-term follow-up confirmed the survival benefit of preoperative chemotherapy, with a $23 \% 5$-year survival rate in the preoperative chemotherapy group compared with $17.1 \%$ in the surgery alone group ( $\mathrm{HR}=0.84 ; 95 \% \mathrm{CI}, 0.72-0.98$; $P=.03){ }^{80,81}$ The Medical Research Council OEO5 trial compared preoperative chemotherapy with 2 cycles of fluorouracil and cisplatin to 4 cycles of epirubicin, oxaliplatin, and capecitabine (ECX) followed by surgery in 897 patients with lower esophageal or EGJ adenocarcinoma. Although a trend toward prolonged PFS and DFS was found with ECX, this did not translate into an OS benefit. ${ }^{53}$ Furthermore, ECX was associated with higher toxicity than fluorouracil and cisplatin $(47 \%$ vs $30 \%$ grade 3-4 toxicities; $P<.001$ ). Therefore, the panel recommends preoperative chemotherapy with fluorouracil and cisplatin for adenocarcinoma of the thoracic esophagus or EGJ (category 2B).

\section{Definitive Chemoradiation Therapy}

Given the efficacy and safety of combined paclitaxel and carboplatin as a preoperative chemoradiation regimen as reported in the CROSS trial, ${ }^{38}$ the NCCN Panel also recommends this regimen as a preferred option for definitive chemoradiation. In a retrospective comparison, definitive chemoradiation with paclitaxel and carboplatin resulted in superior OS, disease-specific survival, locoregional control, and palliation in patients with unresectable esophageal cancer compared with cisplatin and irinotecan. ${ }^{83}$ The FOLFOX regimen and combined fluorouracil and cisplatin have also been proven as effective definitive chemoradiation regimens in clinical trials.

The efficacy of chemoradiation therapy with fluorouracil and cisplatin versus RT alone, each without resection, was studied in an early randomized trial (RTOG 85-01). ${ }^{84,85}$ Compared with patients who received RT alone, patients who received chemoradiation showed a significant improvement in both median survival (14 vs 9 months) and 5 -year OS (27\% vs $0 \%)$ with projected 8-year and 10 -year survival rates of $22 \%$ and $20 \%$, respectively. The incidence of local failure as the first site of failure (defined as local persistence plus recurrence) was also lower in the chemoradiation arm $(47 \%$ vs $65 \%$ in the RT alone arm).

In a randomized phase III trial (PRODIGE5/ACCORD17), 267 patients with unresectable esophageal cancer or who were medically unfit for surgery were randomized to receive definitive chemoradiation with either FOLFOX or 
PRINCIPLES OF SYSTEMIC THERAPY

\begin{tabular}{|c|}
\hline $\begin{array}{l}\text { Preoperative Chemoradiation } \\
\text { (Infusional fluorouracil }{ }^{\mathrm{a}} \text { can be replaced with capecitabine) }\end{array}$ \\
\hline $\begin{array}{l}\text { Preferred Regimens } \\
\text { - Paclitaxel and carboplatin (category } 1)^{1} \\
\left.\text { - Fluorouracil }{ }^{\mathrm{a}} \text { and oxaliplatin (category } 1\right)^{2,3}\end{array}$ \\
\hline $\begin{array}{l}\text { Other Recomended Regimens } \\
\text { Fluorouracil and cisplatin (category } 1)^{4,5} \\
\text { - Irinotecan and cisplatin (category 2B) } \\
\text { - Paclitaxel and fluoropyrimidine } \\
\text { (fluorouracil or capecitabine) (category 2B) }^{7}\end{array}$ \\
\hline
\end{tabular}

\begin{tabular}{|l|}
\hline $\begin{array}{l}\text { Perioperative Chemotherapy } \\
\text { (Only for adenocarcinoma of the thoracic esophagus or EGJ) }\end{array}$ \\
\hline $\begin{array}{l}\text { Preferred Regimens } \\
\text { - Fluoropyrimidine and oxaliplatin }\end{array}$ \\
- Fluorouracil, \\
${\text { (category } 1)^{\text {c }}}^{\text {(ceucovorin, oxaliplatin, and docetaxel (FLOT) }}{ }^{8}$ \\
\hline Other Recomended Regimens \\
- Fluorouracil and cisplatin (category 1$)^{9}$ \\
\hline
\end{tabular}

\section{Preoperative Chemotherapy}

(Only for adenocarcinoma of the thoracic esophagus or EGJ)

- Fluorouracil and cisplatin (category 2B) ${ }^{10}$

aLeucovorin is indicated with certain fluorouracil-based regimens. Dependin

information regarding the leucovorin shortage, please see Discussion.

bThe use of this regimen and dosing schedules is based on extrapolations from published literature and clinical practice.

cDue to toxicity, three-drug regimens are recommended only in select patients who are medically fit.

dCisplatin may not be used interchangeably with oxaliplatin in this setting.

The selection, dosing, and administration of anticancer agents and the management of associated toxicities are complex. Modifications of drug dose and schedule and initiation of supportive care interventions are often necessary because of expected toxicities and because of individual patient variability, prior treatment, nutritional status, and comorbidity. The optimal delivery of anticancer agents therefore requires a health care delivery team experienced in the use of anticancer agents and the management of associated toxicities in patients with cancer. fluorouracil and cisplatin. ${ }^{57}$ The median PFS was 9.7 months in the FOLFOX group compared with 9.4 months in the fluorouracil and cisplatin group $(P=.64) .{ }^{57}$ Although definitive chemoradiation with FOLFOX was not associated with a PFS benefit compared with fluorouracil and cisplatin, the investigators suggest that FOLFOX might be a more convenient option for patients with localized esophageal cancer who may not be candidates for surgery. After a 6-month follow-up, an updated analysis revealed no significant differences in health-related quality of life between patients receiving definitive chemoradiation with FOLFOX versus those receiving fluorouracil and cisplatin. ${ }^{86}$ Therefore, FOLFOX and fluorouracil plus cisplatin are both category 1 preferred recommendations for definitive chemoradiation, although FOLFOX is associated with less treatment-related adverse events.

\section{Postoperative Chemotherapy}

The value of postoperative chemotherapy in treating resectable esophageal and EGJ cancers remains uncertain because phase III randomized controlled trials demonstrating a survival benefit are lacking. Therefore, the regimen listed in the NCCN Guidelines for postoperative chemotherapy (capecitabine and oxaliplatin) is derived from the phase III CLASSIC trial involving patients with stage II or IIIB gastric cancer. ${ }^{56,87}$ In this study, patients were randomized to receive either gastrectomy with D2 lymph node dissection alone $(\mathrm{n}=515)$ or gastrectomy with $\mathrm{D} 2$ lymph node dissection followed by postoperative chemotherapy $(n=520)$. After a median follow-up of 34.2 months, postoperative chemotherapy with capecitabine and oxaliplatin significantly improved 3-year DFS (74\%) compared with surgery alone $(59 \%)$ for all disease stages $(P<.0001){ }^{87}$ After a median follow-up of 62.4 months, the estimated 5 -year DFS rate was $68 \%$ for the postoperative chemotherapy group compared with $53 \%$ for the surgery alone group; the corresponding estimated 5-year OS rates were $78 \%$ and $69 \%$, respectively. ${ }^{56}$ Based on these data, the panel recommends postoperative capecitabine and oxaliplatin to patients with resectable esophageal or EGJ cancers.

\section{Systemic Therapy for Locally Advanced or Metastatic Disease}

\section{First-Line Therapy}

Systemic therapy can provide palliation of symptoms, improved survival, and enhanced quality of life in patients with locally advanced or metastatic esophageal or EGJ cancers. ${ }^{88-90}$ First-line systemic therapy regimens 
PRINCIPLES OF SYSTEMIC THERAPY

Systemic Therapy for Unresectable Locally Advanced, Recurrent or Metastatic Disease (where local therapy is not indicated

- Trastuzumab should be added to first-line chemotherapy for HER2 overexpressing metastatic adenocarcinoma

(See Principles of Pathologic Review and Biomarker Testing [ESOPH-B])

- Combination with fluoropyrimidine and cisplatin (category 1$)^{17}$

- Combination with other chemotherapy agents (category 2B)

- Trastuzumab is not recommended for use with anthracyclines

First-Line Therapy

- Two-drug cytotoxic regimens are preferred because of lower toxicity.

- Three-drug cytotoxic regimens should be reserved for medically fit patients with good PS and access to frequent toxicity evaluation.

Preferred Regimens

- Fluoropyrimidine (fluorouracil ${ }^{\mathrm{a}}$ or capecitabine) and oxaliplatin ${ }^{18-20}$

- Fluoropyrimidine (fluorouracil ${ }^{\mathrm{a}}$ or capecitabine) and cisplatin ${ }^{18,21-23}$ (category 2B)

Other Recommended Regimens

- Paclitaxel with cisplatin or carboplatin ${ }^{24-26}$

- Docetaxel with cisplatin 27,28

- Fluoropyrimidine $e^{20,29,30}$ (fluorouracil ${ }^{\mathrm{a}}$ or capecitabine)

- Docetaxel ${ }^{31,32}$

- Paclitaxel 33,34

- Fluorouracil ${ }^{\mathrm{a}, \mathrm{e}}$ and irinotecan ${ }^{35}$

- DCF modifications

- Docetaxel, cisplatin, and fluorouracil ${ }^{a, 36}$

- Docetaxel, oxaliplatin, and fluorouracil ${ }^{37}$

- Docetaxel, carboplatin, and fluorouracil (category 2B) ${ }^{38}$

- ECF (epirubicin, cisplatin, and fluorouracil) (category 2B) ${ }^{39}$

- ECF modifications (category 2B) (0,41 $^{4}$

- Epirubicin, oxaliplatin, and fluorouracil

- Epirubicin, cisplatin, and capecitabine

- Epirubicin, oxaliplatin, and capecitabine

aLeucovorin is indicated with certain fluorouracil-based regimens. Depending on availability, these regimens may be used with or without leucovorin. For important

information regarding the leucovorin shortage, please see Discussion.

eCapecitabine cannot be used interchangeably with fluorouracil in regimens containing irinotecan

with 2 cytotoxic drugs are preferred for patients with advanced disease because of their lower toxicity. Threedrug cytotoxic regimens should be reserved for medically fit patients with good performance status and access to frequent toxicity evaluation. Based on the results of the ToGA trial, the guidelines recommend the addition of trastuzumab to first-line chemotherapy for patients with HER2-positive metastatic adenocarcinoma (category 1 for combination with cisplatin and fluoropyrimidine; category $2 \mathrm{~B}$ for combination with other chemotherapy agents). ${ }^{26}$ The use of trastuzumab in combination with anthracyclines is not recommended. See "Targeted Therapies" (page 873) for more information on trastuzumab.

The preferred regimens for first-line systemic therapy include a fluoropyrimidine (fluorouracil or capecitabine) combined with either oxaliplatin ${ }^{91-93}$ or cisplatin (category 2B) ${ }^{91,94-96}$ (see ESOPH-F page 3 of 13, above). A phase III trial conducted by the German Study Group compared treatment with fluorouracil and cisplatin to FOLFOX in patients $(n=220)$ with previously untreated advanced adenocarcinoma of the stomach or EGJ. ${ }^{91}$ Results showed that FOLFOX (referred to as FLO) was associated with significantly less toxicity and showed a trend toward improved median PFS (5.8 vs 3.9 months; $P=.77$ ) compared with fluorouracil and cisplatin (FLP). However, no significant difference was seen in median OS (10.7 vs 8.8 months, respectively) between the 2 groups. Interestingly, FOLFOX resulted in significantly superior response rates $(41.3 \%$ vs $16.7 \% ; P=.12$ ), time to treatment failure (5.4 vs 2.3 months; $P<.001$ ), PFS (6.0 vs 3.1 months; $P=.029$ ), and improved OS (13.9 vs 7.2 months) compared with FLP in patients $>65$ years $(n=94)$. Therefore, FOLFOX offers reduced toxicity and similar efficacy compared with fluorouracil plus cisplatin and may also be associated with improved efficacy in older adult patients.

Recommendations for the use of regimens combining a platinum agent with capecitabine as first-line therapy for advanced or metastatic esophageal or EGJ cancers have been extrapolated from trials involving patients with advanced gastric cancer. ${ }^{93,96-98}$ A phase III randomized trial (ML 17032) that evaluated the efficacy of combined capecitabine and cisplatin (XP) compared with fluorouracil and cisplatin (FP) found that XP was noninferior to FP as first-line therapy in patients with advanced gastric cancer. ${ }^{96}$ Additionally, 2 phase II trials concluded that capecitabine in combination with oxaliplatin is active and well-tolerated as first-line therapy for advanced gastric cancer. ${ }^{97,98}$ Furthermore, results of a meta-analysis suggest that OS was superior in patients with advanced gastroesophageal cancer treated 
PRINCIPLES OF SYSTEMIC THERAPY

Systemic Therapy for Unresectable Locally Advanced, Recurrent or Metastatic Disease (where local therapy is not indicated)

\begin{tabular}{|c|}
\hline $\begin{array}{l}\text { Second-Line or Subsequent Therapy } \\
\text { - Dependent on prior therapy and PS }\end{array}$ \\
\hline $\begin{array}{l}\text { Preferred Regimens } \\
\text { - Ramucirumab and paclitaxel for adenocarcinoma } \\
\text { (category } 1 \text { for EGJ adenocarcinoma; category } 2 \mathrm{~A} \text { for esophageal adenocarcinoma) } \\
\text { - Docetaxel (category } 1)^{31,32} \\
\text { - Paclitaxel (category } 1)^{33,34,43} \\
\text { - Irinotecan (category } 1)^{43-46} \\
\text { - Trifluridine and tipiracil for EGJ adenocarcinoma (category } 1)^{47} \\
\text { - For third-line or subsequent therapy } \\
\text { - Fluorouracila,e and irinotecan } 44,48,49 \\
\text { - Pembrolizumab } \\
\text { - For second-line or subsequent therapy for MSI-H or dMMR tumors }\end{array}$ \\
\hline $\begin{array}{l}\text { Other Recommended Regimens } \\
\text { - Ramucirumab for adenocarcinoma (category } 1 \text { for EGJ adenocarcinoma; category } 2 A \text { for esophageal adenocarcinoma) } \\
\text { - Irinotecan and cisplatin } 19,53 \\
\text { - Pembrolizumab } \\
\text { - For second-line therapy for esophageal squamous cell carcinoma, esophageal adenocarcinoma and EGJ adenocarcinoma with PD-L1 } \\
\text { expression levels by CPS of } \geq 10 \text { (category } 2 B)^{54} \\
\text { - For third-line or subsequent therapy for esophageal and EGJ adenocarcinoma with PD-L1 expression levels by CPS of } \geq 1^{\mathrm{f}, 55} \\
\text { - Docetaxel and irinotecan (category } 2 \mathrm{~B})^{56}\end{array}$ \\
\hline
\end{tabular}

Docetaxel and irinotecan (category $2 \mathrm{~B})^{56}$

\footnotetext{
aLeucovorin is indicated with certain fluorouracil-based regimens. Depending on availability, these regimens may be used with or without leucovorin. For important information regarding the leucovorin shortage, please see Discussion.

e Capecitabine cannot be used interchangeably with fluorouracil in regimens containing irinotecan

fPembrolizumab is approved for the third-line treatment of patients with EGJ adenocarcinoma with PD-L1 expression levels by CPS of $\geq 1$, as determined by an FDA-approved companion diagnostic test. The NCCN Panel recommends that this pembrolizumab treatment option be extended to patients with esophageal adenocarcinomas with PD-L1 expression levels by CPS of $\geq 1$. For more information on PD-L1 testing, See Principles of Pathology and Biomarker Testing (ESOPH-B).

with capecitabine-based combinations compared with patients treated with fluorouracil-based combinations, although no significant difference in PFS between treatment groups was seen. ${ }^{99}$ These results suggest that capecitabine can be considered an effective alternative to fluorouracil in the treatment of patients with advanced gastroesophageal cancers.

Docetaxel, cisplatin, and fluorouracil (DCF) has also demonstrated activity in patients with locally advanced or metastatic gastroesophageal cancer. ${ }^{100,101}$ An international phase III study (V325) that randomized 445 patients with untreated advanced gastric or EGJ cancer to receive either DCF or cisplatin and fluorouracil (CF) found that the addition of docetaxel to CF significantly improved time to progression, OS, and overall response rate (ORR) ${ }^{101}$ However, DCF was associated with increased toxicities, including myelosuppression and infectious complications. ${ }^{101}$ Various modifications of the DCF regimen have shown improved safety in clinical trials of patients with advanced gastroesophageal cancer compared with the DCF regimen evaluated in the V325 study. ${ }^{102-105}$ In a randomized phase II study, a dose-modified DCF regimen was less toxic than standard DCF and was also associated with improved median OS (18.8 vs 12.6 months; $P=.007$ ) in previously untreated patients with metastatic gastric or EGJ adenocarcinoma. ${ }^{103}$ In another randomized phase II trial that evaluated docetaxel plus oxaliplatin with or without infusional fluorouracil or capecitabine in patients with metastatic or locally recurrent gastric or EGJ adenocarcinoma, docetaxel, oxaliplatin, and fluorouracil had a better safety profile and was associated with higher response rates and longer median PFS and OS (47\%, 7.7 months, and 14.6 months, respectively) compared with docetaxel and oxaliplatin $(23 \%, 4.5$ months, and 9 months, respectively) or docetaxel, oxaliplatin, and capecitabine $(26 \%$, 5.6 months, and 11.3 months, respectively). ${ }^{102}$ Additionally, the frequency of grade 3-4 toxicities was lower among patients treated with docetaxel, oxaliplatin, and fluorouracil $(25 \%)$ compared with those treated with docetaxel and oxaliplatin $(37 \%)$ or docetaxel, oxaliplatin, and capecitabine $(38 \%)$. Therefore, due to concerns regarding toxicity, dose-modified DCF or other DCF modifications should be used as alternative options to the standard DCF regimen for first-line therapy.

First-line treatment with irinotecan-based regimens has been explored extensively in clinical trials involving patients with advanced or metastatic gastroesophageal cancers. ${ }^{106-112}$ The results of a randomized phase III study comparing FOLFIRI (fluorouracil and irinotecan) to CF in 
1van Hagen P, Hulshof MC, van Lanschot JJ, et al. Preoperative
chemoradiotherapy for esophageal or junctional cancer. N Engl chemoradiotherapy for esop

Conroy T, Galais MP, Raoul JL, et al. Definitive

chemoradiotherapy with FOLFOX versus fluorouracil and cisplatin in patients with oesophageal cancer (PRODIGE5/ ACCORD17): final results of a rand

Khushalani NI Leichman CG Proulx G, et al Oxaliplatin in combination with protracted-infusion fluorouracil and radiation: report of a clinical trial for patients with esophageal cancer. $J$ Clin Oncol 2002;20:2844-2850.

${ }^{4}$ Tepper J, Krasna MJ, Niedzwiecki D, et al. Phase III trial of

trimodality therapy with cisplatin, fluorouracil, radiotherapy, an surgery compared with surgery alone for esophageal cancer: CALGB 9781. J Clin Oncol 2008;26:1086-1092.

Bedenne L, Michel P, Bouche O, et al. Chemoradiation

followed by surgery compared with chemoradiation alone in

squamous cancer of the esophagus: FFCD 9102. J Clin Onco

2007;25:1160-1168.

, al A single institution experience with neoadjuvant chemoradiation (CRT) with catecan (I) and cisplatin (C) in locally advanced esophag 15):Abstract e15619.

Ajani JA, Winter K, Okawara GS, et al. Phase II trial of preoperative chemoradiation in patients with localized gastric adenocarcinoma (RTOG 9904): quality of combined modality therapy and pathologic response. J Clin Oncol 2006;24:3953 3958 .
$8 A$ Al-Batran S-E, Homann N, Pauligk C, et al. Perioperative chemotherapy with fluorouracil plus leucovorin, oxaliplatin, and docetaxel versus fluorouracil or capecitabine plus cisplatin an epirubicin for locally advanced, resectable gastric or gastrooesophageal junction adenocarcinoma (FLOT4): a randomised phase 2/3 trial. Lancet 2019

${ }^{9}$ Ychou M, Boige V, Pignon J-P, et al. Perioperative

chemotherapy compared with surgery alone for resectable gastroesophageal adenocarcinoma: an FNCLCC and FFCD multicenter phase III trial. J Clin Oncol 2011:29:1715-1721.
PRINCIPLES OF SYSTEMIC THERAPY REFERENCES

${ }^{10}$ Alderson D, Cunningham D, Nankivell M, et al. Neoadjuvan cisplatin and fluorouracil versus epirubicin, cisplatin, and capecitabine followed by resection in patients with oesophage denocarcinoma (UK MRC OE05): an open-label, randomised phase 3 trial. Lancet Oncol 2017;18:1249-1260.

Minsky BD, Pajak TF, Ginsberg RJ, et al. INT 0123 (Radiation Therapy Oncology Group 94-05) phase III trial of combinedmodality therapy or esophageal cancer: high-dose versus

1174 .
12 Urba SG, Orringer MB, lanettonni M, et al. Concurrent

cisplatin, paclitaxel, and radiotherapy as preoperative treatme for patients with locoregional esophageal carcinoma. Cancer 2003;98:2177-2183

${ }^{\mathrm{L}} \mathrm{Li} Q$ Q, Liu MZ, Hu YH, et al. Definitive concomitant chemoradiotherapy with docetaxel and cisplatin in squamous esophageal carcinoma. Dis Esophagus 2010;23:253-259. Day FL, Leong T, Ngan S, et al. Phase I trial of docetax cisplatin and concurrent radical radiotherapy in locally advance oesophageal cancer. Br J Cancer 2011;104:265-27 . ISmalley SR, Benedetti JK, Haller DG, et al. Updated analysis adjunt redion gantric cancer resection. J Clin Oncol 2012:30:2327-2333. ${ }_{16}$ Noh SH Park SR Yang HK oxaliplatin for gastric cancer after D2 gastrectomy (CLASSIC) 5-year follow-up of an open-label, randomised phase 3 trial. Lancet Oncol 2014: 15:1389-1396.

17 Bang YJ, Van Cutsem E, Feyereislova A, et al. Trastuzumab in combination with chemotherapy versus chemotherapy alon for treatment of HER2-positive advanced gastric or gastrooesophageal junction cancer (ToGA): a phase 3, open-label, randomised controlled trial. Lancet 2010;376:687-697. Al-Batran S-E, Hartmann JT, Probst S, et al. Phase III trial in metastatic gastroesophageal adenocarcinoma with fluorourac leucovorin plus either oxaliplatin or cisplatin: a study of the 2008;26:1435-1442

2008,26:1435-1442 3 B Niedzwiecki D, et al CALGB 80403 (Alliance)/E1206: a randomized phase Il study of hree chemotherpy regimens plus ceturimab in metastatic esophageal and gastroesophageal junction cancers. J Clin Oncol 2016;34:2736-2742.
${ }^{20}$ Kim GM, Jeung HC, Rha SY, et al. A randomized phase II trial of S-1-oxaliplatin versus capecitabine-oxaliplatin in advanced gastric cancer. Eur J Cancer 2012,48.518-526. Lorenzen S, Schuster T, Porschen R, et al. Cetuximab plus cisplatin-5-fluorouracil versus cisplatin-5-fluorouracil alone in first-line mitastatic squandus cell carchoma of the esophagus: a randomized phase II study of the Arbeitsgemeinschaft 22Bouche O Raoul Jl, Bonnetain $F$ et al, Randomized multicenter phase II trial of a biweekly regimen of fluorourac and leucovorin (LV5FU2) LV5FU2 plus cisplatin, or LV5FU2 plus irinotecan in patients with previously untreated metastatic gastric cancer: a Federation Francophone de Cancerologie Digestive Group Study-FFCD 9803. J Clin Oncol 2004;22:4319 4328.

${ }^{23}$ Kang YK, Kang WK, Shin DB, et al. Capecitabine/cisplatin versus 5 -fluorouracil/cisplatin as first-line therapy in patients with advanced gastric cancer: a randomised phase

woninferiority trial. Ann Oncol 2009,20:666-673.

(1) of parcinoma of 25 cisplatin and paclitaxel in patients with locally adverapy with recurrent or metastatic oesophageal cancer. Br J Cancer 1998:78:511-514

${ }^{26}$ Gadgeel SM, Shields AF, Heilbrun LK, et al. Phase II study of paclitaxel and carboplatin in patients with advanced gastric

${ }^{27}$ Ajani JA, Fodor MB, Tjulandin SA, et al. Phase II multiinstitutional randomized trial of docetaxel plus cisplatin with or without fluorouracil in patients with untreated, advanced gastric, or gastroesophageal adenocarcinoma. J Clin Oncol

8 Kim JY, Do YR, Park KU, et al. A multi-center phase II study wiscetaxel plus cisplatin as first-line therapy in patients Chemother Pharmacol 2010;66:31-36.

${ }^{29}$ Ohtsu A, Shimada Y, Shirao K, et al. Randomized phase III tria of fluorouracil alone versus fluorouracil plus cisplatin versus uracil and tegafur plus mitomycin in patients with unresectable, advanced gastric cancer: The Japan Clinical Oncology Group Study (JCOG9205). J Clin Oncol 2003;21:54-59. patients with advanced gastric or EGJ adenocarcinoma $(n=337)$ showed that FOLFIRI was noninferior to $\mathrm{CF}$ in terms of PFS (PFS at 6 and 9 months were 38\% and $20 \%$, respectively, for FOLFIRI compared with $31 \%$ and $12 \%$, respectively, for $\mathrm{CF}$ ) but not in terms of OS (9 vs 8.7 months) or time to progression (5 vs 4.2 months). ${ }^{107}$ FOLFIRI was also associated with a more favorable toxicity profile. Therefore, the NCCN Panel believes that FOLFIRI is an acceptable first-line therapy option for patients with advanced or metastatic esophageal or EGJ adenocarcinoma. Other recommended regimens for first-line therapy include paclitaxel with either cisplatin or carboplatin, ${ }^{113-115}$ docetaxel with cisplatin, ${ }^{100,116}$ or singleagent fluoropyrimidine (fluorouracil or capecitabine), ${ }^{95,117,118}$ docetaxel, ${ }^{88,119}$ or paclitaxel. ${ }^{120,121}$ Combined docetaxel, carboplatin, and fluorouracil ${ }^{105}$ as well as ECF ${ }^{122}$ and ECF modifications ${ }^{123,124}$ are category $2 \mathrm{~B}$ recommendations in this setting.

\section{Second-Line and Subsequent Therapy}

The selection of regimens for second-line or subsequent therapy depends on prior therapy and performance status (see ESOPH-F page 4 of 13, page 870). Based on the available data and FDA approvals, the guidelines have included the targeted therapy ramucirumab (category
1 for EGJ adenocarcinoma; category 2A for esophageal adenocarcinoma) as a single agent or in combination with paclitaxel (preferred) as treatment options for second-line or subsequent therapy. ${ }^{125,126}$ Pembrolizumab has been included as a preferred second-line or subsequent therapy option for MSI-H/dMMR tumors. ${ }^{32,127}$ Pembrolizumab has also been included as a second-line therapy option for esophageal cancers with PD-L1 expression levels by CPS of $\geq 10$ (category $2 \mathrm{~B})^{37}$ and as a third-line or subsequent therapy option for esophageal and EGJ adenocarcinomas with PD-L1 expression levels by CPS of $\geq 1 .{ }^{36}$ See "Targeted Therapies" (page 873) for more information on ramucirumab and pembrolizumab.

Category 1 preferred options for second-line or subsequent therapy include single-agent docetaxel, ${ }^{88,119}$ paclitaxel, ${ }^{120,121,128}$ and irinotecan..$^{89,128-130}$ In a randomized phase III trial (COUGAR-02), single-agent docetaxel was shown to significantly increase 12-month OS compared with active symptom control alone (5.2 vs 3.6 months, respectively; $\mathrm{HR}=0.67 ; P=.01) .{ }^{88}$ Additionally, patients receiving docetaxel reported less pain, nausea, vomiting, dysphagia, and constipation. A randomized phase III trial comparing second-line therapy with paclitaxel to irinotecan in patients with advanced gastric 


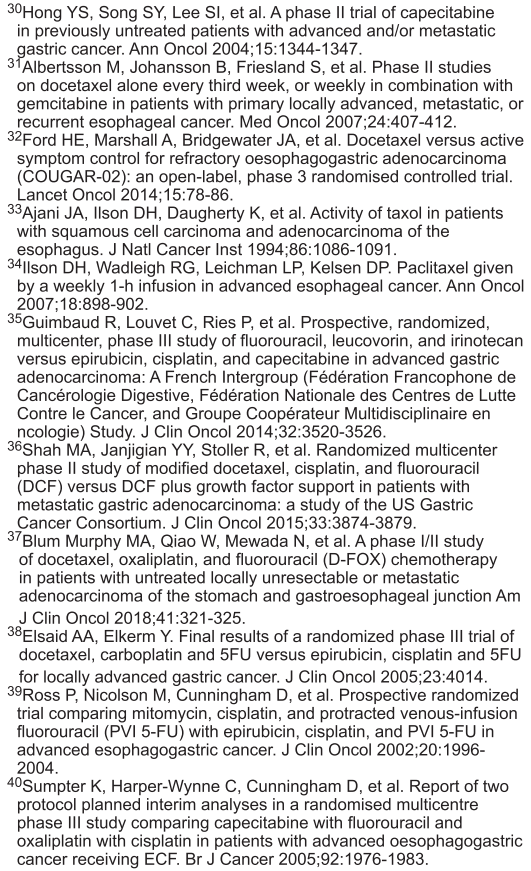

PRINCIPLES OF SYSTEMIC THERAPY REFERENCES

${ }^{41}$ Cunningham D, Starling N, Rao S, et al. Capecitabine and oxaliplatin for advanced esophagogastric cancer. N Engl J Med 2008;358:36-46.

Wike $H$, Muro K, Van Cutsem E, et al. Ramucirumab plus paclit versus placebo plus paclitaxel in patients with previously treated advanced gastric or gastro-oesophageal junction adenocarcinoma 2014:1224-1235.

${ }^{43}$ Hironaka S, Ueda S, Yasui H, et al. Randomized, open-label, phase III study comparing irinotecan with paclitaxel in patients with advanced gastric cancer without severe peritoneal metastasis after fallure of prior combination chemotherapy using fluoropyrimidine

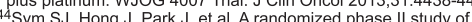
iweekly irinotecan monotherapy or a combination of irindy of plus 5 -fluorouracil/leucovorin (mFOLFIRI) in patients with metasta gastric adenocarcinoma refractory to or progressive after first-line chemotherapy. Cancer Chemother Pharmacol 2013;71:481-488. Thuss-Paltence PC, KretzschmarA, Bichev D, et al. Survival advantage for irinotecan versus best supportive care as second-lin Arbeitsgemeinschaft Internistische Onkologie (AIO). Eur J Cancer 2011;:47:2306-2314.

${ }^{46}$ Fuchs CS, Moore MR, Harker G, et al. Phase III comparison of two irinotecan dosing regimens in second-line therapy of metasta colorectal cancer. J Clin Oncol 2003;21:807-814.
${ }^{47}$ Shitara K, Doi T, Dvorkin M, et al. Trifluridine/tipiracil versus placebo in patients with heavily pretreated metastatic gastric cancer placebo in patients with heavily pretreated metastatic gastric canc
(TAGS): a randomised, double-blind, placebo-controlled, phase 3 trial. Lancet Oncol 2018:19:1437-1448.

${ }^{48}$ Sym SJ, Ryu MH, Lee JL, et al. Salvage chemotherapy with biweekly irinotecan, plus 5-fluorouracil and leucovorin in patients with advanced gastric cancer previously treated with fluoropyrimidine, platinum, and taxane. Am J Clin Oncol 2008;31:151-156.

4 Assersohn L, Brown G, Cunningham D, et al. Phase Il study of irinotecan and 5 -fluorouracil/leucovorin in patients with primary refractory or relapsed advanced oesophageal and gastric

carcinoma. Ann Oncol 2004;15:64-69.
50 Le DT. Uram JN Mismatch-Repair Deficiency N N Eng- I Med 2015;372:2509-2520. DT Durham JN Smith KN et al. Mismatch repair deficiency predicts response

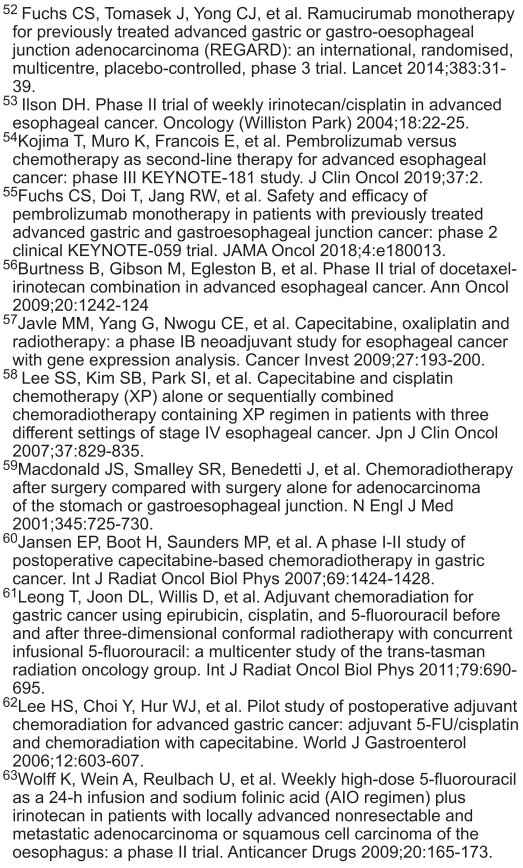

cancer found similar OS between the 2 groups ( 9.5 months in the paclitaxel group vs 8.4 months in the irinotecan group; $\mathrm{HR}=1.13 ; P=.38) .{ }^{128}$ Therefore, single-agent docetaxel, paclitaxel, and irinotecan are all recommended as preferred second-line treatment options for advanced gastroesophageal cancers.

Second-line therapy with FOLFIRI has also been shown to be active and well-tolerated in patients with metastatic gastroesophageal cancers. ${ }^{129,131,132}$ A phase II trial investigating the efficacy and toxicity of FOLFIRI in patients $(n=40)$ with refractory or relapsed esophageal or gastric cancer reported an ORR of $29 \%$ and median OS of 6.4 months. Another phase II trial reported similar results with an ORR of $20 \%$ and OS of 6.7 months in patients with advanced gastric cancer $(n=59)$ treated with FOLFIRI in the second-line setting. ${ }^{129}$ Additionally, FOLFIRI was shown to be an effective and safe treatment option in a cohort of patients with metastatic gastric or EGJ cancers refractory to docetaxel-based chemotherapy. ${ }^{133}$ In this study, the ORR was $22.8 \%$ and median PFS and OS were 3.8 and 6.2 months, respectively. The most common grade $3-4$ toxicities were neutropenia (28.5\%) and diarrhea (14.5\%). Therefore, FOLFIRI is considered as a preferred treatment option that can be safely used in the second-line setting if it was not previously used in first-line therapy. Other recommended combined regimens for second-line therapy include irinotecan and cisplatin ${ }^{92,106}$ and irinotecan and docetaxel (category 2B). ${ }^{109}$

A recently published phase III trial (TAGS) has shown activity for the combined regimen of trifluridine and tipiracil in metastatic gastric and EGJ adenocarcinoma in the third-line setting. ${ }^{134}$ The trifluridine and tipiracil regimen, which was approved by the FDA in 2019 for previously treated recurrent or metastatic gastric and EGJ adenocarcinoma, ${ }^{135}$ was initially investigated in a phase II trial in Japan which reported a median OS of 8.7 months and a disease control rate of $65.5 \%{ }^{136} \mathrm{In}$ the global phase III TAGS trial, 507 patients with heavily pretreated metastatic gastric or EGJ cancer were randomized 2:1 to receive trifluridine and tipiracil plus best supportive care $(n=337)$ or placebo plus best supportive care $(n=170) .{ }^{134}$ This study reported a significant improvement in median OS by 2.1 months (5.7 vs 3.6 months) with the trifluridine and tipiracil regimen compared with placebo ( $\mathrm{HR}=0.69 ; 95 \% \mathrm{CI}, 0.56-0.85$; $P=.0003)$. PFS was statistically significantly longer in the trifluridine and tipiracil group (2.0 vs 1.7 months; $\mathrm{HR}=0.57 ; 95 \% \mathrm{CI}, 0.47-0.70 ; P<.0001)$. The most frequently reported grade $3-4$ toxicities associated with the 
trifluridine and tipiracil regimen were neutropenia (38\%), leukopenia (21\%), anemia (19\%), and lymphocytopenia (19\%), which was consistent with other studies involving these agents. Trifluridine and tipiracil is recommended as a preferred category 1 treatment option for patients with recurrent or metastatic EGJ adenocarcinoma in the third-line or subsequent setting after prior fluoropyrimidine-, platinum-, taxane-, or irinotecanbased chemotherapy and anti-HER2 therapy (if HER2positive). However, trifluridine and tipiracil did not result in any partial or complete responses and produced substantial grade 3-4 toxicities. Therefore, this treatment should be considered for a very select population of patients with low-volume EGJ adenocarcinoma who have minimal or no symptoms and the ability to swallow pills. Other recommended regimens for thirdline or subsequent therapy for esophageal and EGJ cancers include regimens recommended for second-line therapy that were not previously used and pembrolizumab for adenocarcinomas with PD-Ll expression levels by CPS of $\geq 1$.

\section{Targeted Therapies}

At present, 3 targeted therapeutic agents, trastuzumab, ramucirumab, and pembrolizumab, have been approved by the FDA for use in esophageal and EGJ cancers. ${ }^{31,35,137-139}$ Treatment with trastuzumab is based on testing for HER2 status. ${ }^{26}$ Treatment with pembrolizumab is based on testing for microsatellite instability and/or PD-L1 expression. . $^{32,36,127,140}$

\section{Trastuzumab}

The ToGA trial was the first randomized, prospective, multicenter, phase III trial that evaluated the efficacy and safety of trastuzumab in HER2-positive advanced gastric and EGJ adenocarcinoma. ${ }^{26}$ In this trial, 594 patients with HER2-positive, locally advanced, recurrent, or metastatic gastric or EGJ adenocarcinoma were randomized to receive trastuzumab plus chemotherapy (cisplatin plus fluorouracil or capecitabine) or chemotherapy alone. ${ }^{26}$ The majority of patients had gastric cancer $(80 \%$ in the trastuzumab group and $83 \%$ in the chemotherapy group). Median follow-up time was 19 months and 17 months, respectively, in the 2 groups. Results showed significant improvement in median OS with the addition of trastuzumab to chemotherapy in patients with HER2-positive disease (13.8 vs11 months, respectively; $P=.046$ ). This study established trastuzumab in combination with cisplatin and a fluoropyrimidine as the standard treatment of patients with HER2-positive metastatic gastroesophageal adenocarcinoma. The addition of trastuzumab was particularly beneficial in patients with a tumor score of IHC $3+$ or IHC $2+$ and FISH positivity for HER2. In a post hoc subgroup analysis, the addition of trastuzumab to chemotherapy further improved OS in patients whose tumors were IHC $2+$ and FISH positive or IHC $3+(n=446$; 16 vs 11.8 months; $\mathrm{HR}=0.65$ ) compared with those with tumors that were IHC 0 or $1+$ and FISH positive $(n=131$; 10 vs 8.7 months; $\mathrm{HR}=1.07$ ).

In a retrospective study of 34 patients with metastatic gastric or EGJ adenocarcinoma, the combination of trastuzumab with a modified FOLFOX regimen (mFOLFOX6) improved tolerability compared with the cisplatin plus fluorouracil regimen in previously untreated patients with HER2-positive tumors. ${ }^{141}$ The ORR with this regimen was $41 \%$, and median PFS and OS were 9.0 months and 17.3 months, respectively. The most frequent grade $3-4$ toxicities were neutropenia $(8.8 \%)$ and neuropathy (17.6\%). These results suggest that the combination of mFOLFOX6 and trastuzumab is an effective regimen with an acceptable safety profile and warrants further study in patients with HER-2+ gastroesophageal cancers.

\section{Ramucirumab}

Ramucirumab, a VEGFR-2 antibody, has shown favorable results in patients with previously treated advanced or metastatic gastroesophageal cancers in 2 phase III clinical trials. ${ }^{125,126}$ An international randomized multicenter phase III trial (REGARD) demonstrated a survival benefit for ramucirumab in patients with advanced gastric or EGJ adenocarcinoma progressing after firstline chemotherapy. ${ }^{125}$ In this study, 355 patients were randomized to receive ramucirumab $(n=238 ; 178$ had gastric cancer and 60 had EGJ adenocarcinoma) or placebo $(n=117 ; 87$ had gastric cancer and 30 had EGJ adenocarcinoma). Median OS was 5.2 months in patients treated with ramucirumab compared with 3.8 months for those in the placebo group $(P=.047)$. Ramucirumab was associated with higher rates of hypertension than placebo ( $16 \%$ vs $8 \%$ ), whereas rates of other adverse events were similar.

A more recent international phase III randomized trial (RAINBOW) evaluated paclitaxel with or without ramucirumab in patients $(n=665)$ with metastatic gastric or EGJ adenocarcinoma progressing on first-line chemotherapy. ${ }^{126}$ Patients randomized to receive ramucirumab plus paclitaxel $(n=330)$ had significantly longer median OS (9.63 months) compared with patients receiving paclitaxel alone $(\mathrm{n}=335 ; 7.36$ months; $P<.0001)$. The median PFS was 4.4 months and 2.86 months, respectively, for the 2 treatment groups. Additionally, the ORR was $28 \%$ for ramucirumab plus paclitaxel compared with $6 \%$ for paclitaxel alone $(P=.0001)$. However, neutropenia and hypertension were more common with ramucirumab plus paclitaxel. Based on the results of these 2 studies, ramucirumab (as a single agent or in 
combination with paclitaxel) was approved by the FDA for the treatment of patients with advanced gastric or EGJ adenocarcinoma refractory to or progressive after first-line therapy with platinum- or fluoropyrimidinebased chemotherapy. Interestingly, an exposure-response analysis of these trials revealed that ramucirumab was a significant predictor of OS and PFS in both trials. ${ }^{142}$ Higher ramucirumab exposure was associated with longer OS and PFS, but also with higher rates of grade $\geq 3$ hypertension, leukopenia, and neutropenia. This exploratory exposure-response analysis suggests a positive relationship between ramucirumab exposure and efficacy with manageable toxicities.

An international randomized phase III trial (RAINFALL) has recently completed investigation of ramucirumab in combination with a fluoropyrimidine and cisplatin in the first-line treatment of gastroesophageal adenocarcinoma. ${ }^{143}$ This trial randomized 645 patients to receive capecitabine and cisplatin in combination with ramucirumab $(n=326)$ or placebo $(n=319)$. Preliminary results showed that median PFS was significantly longer in patients treated with ramucirumab versus placebo (5.7 vs 5.4 months, respectively; $P=.011 ; \mathrm{HR}=0.75 ; 95 \% \mathrm{CI}$, 0.61-0.94). However, no improvement in median OS was observed with the addition of ramucirumab (11.2 vs 10.7 months; $P=.68$; $\mathrm{HR}=0.96$; $95 \% \mathrm{CI}, 0.80-1.16$ ). The ORR was $41.1 \%$ (95\% CI, 35.8-46.4) in the ramucirumab arm compared with $36.4 \%$ (95\% CI, 31.1-41.6) in the placebo arm. The most common grade $\geq 3$ adverse events in the ramucirumab arm were neutropenia, anemia, and hypertension. These early results suggest that the addition of ramucirumab may not reduce the risk of disease progression or death in treatment-naïve patients with metastatic gastroesophageal adenocarcinoma. Therefore, the addition of ramucirumab to first-line fluoropyrimidine and cisplatin chemotherapy is not recommended at this time. However, more data are needed to ascertain whether the addition of ramucirumab to other firstline chemotherapy regimens can improve OS in these patients.

\section{Pembrolizumab}

Pembrolizumab is a monoclonal PD-1 antibody directed against PD-1 receptors that was granted accelerated approval by the FDA in 2017 for the treatment of patients with unresectable or metastatic MSI-H or dMMR solid tumors that have progressed after previous treatment and who have no satisfactory alternative treatment options. ${ }^{31}$ This first-ever tissue- and site-agnostic approval was based on data from 149 patients with MSI-H/dMMR cancers (90 patients had colorectal cancer) enrolled across 5 multicenter single-arm clinical trials. The ORR was $39.6 \%$ (95\% CI, 31.7-47.9) and responses lasted $\geq 6$ months for $78 \%$ of those who responded to pembrolizumab.
There were 11 complete responses and 48 partial responses to pembrolizumab and the ORR was similar irrespective of whether patients were diagnosed with colorectal cancer $(36 \%)$ or a different cancer type $(46 \%$ across the 14 other cancer types).

One of the trials included in the FDA approval was KEYNOTE-016, a multicenter phase II trial that evaluated the activity of pembrolizumab in 41 patients with metastatic treatment-refractory dMMR colorectal cancers, MMR-proficient colorectal cancers, or dMMR noncolorectal cancers who had received at least 2 previous lines of chemotherapy. ${ }^{32,127}$ In this study, the immune-related ORR for patients with dMMR noncolorectal cancers $(n=9)$ was $71 \%$ with an immune-related PFS rate of $67 \%$ at 20 weeks. ${ }^{127}$ Median PFS was 5.4 months and OS was not reached. Adverse events of clinical interest included rash or pruritus $(24 \%)$, thyroid dysfunction $(10 \%)$, and asymptomatic pancreatitis (15\%). These events were similar to those reported in other trials involving pembrolizumab. In a recently reported expansion of this study, data from 86 patients with dMMR tumors representing 12 different cancer types, including gastroesophageal cancers, attained an ORR of $53 \%$, with $21 \%$ of patients experiencing a complete response. ${ }^{32}$ Although median PFS and OS have not yet been reached, estimates of these outcomes at 1 and 2 years are $64 \%$ and $53 \%$ for PFS and $76 \%$ and $64 \%$ for OS, respectively. The KEYNOTE016 trial is still recruiting patients at several institutions (ClinicalTrials.gov identifier: NCT01876511).

Another 2017 FDA approval for pembrolizumab was for the treatment of patients with recurrent, locally advanced, or metastatic PD-L1-positive (CPS $\geq 1$ ) gastric or EGJ adenocarcinoma who have progressed after 2 or more prior lines of therapy, including fluoropyrimidineand platinum-containing chemotherapy and, if appropriate, HER2-targeted therapy. ${ }^{35}$ This approval was based on the results of 2 KEYNOTE studies (KEYNOTE-012 and KEYNOTE-059). KEYNOTE-012 was a multicenter, phase Ib study that evaluated the safety and activity of pembrolizumab in patients with PD-L1-positive recurrent or metastatic gastric or EGJ adenocarcinoma. ${ }^{144}$ The ORR was $22 \%$, and $13 \%$ of patients had grade $3-4$ treatmentrelated adverse events including fatigue, pemphigoid, hypothyroidism, peripheral sensory neuropathy, and pneumonitis. The results of this trial justified the study of pembrolizumab monotherapy in cohort 1 of the phase II KEYNOTE-059 trial, which included 259 patients with gastric or EGJ adenocarcinoma who had progressed on $\geq 2$ prior lines of therapy. ${ }^{36}$ Of those with PD-L1-positive tumors $(57.1 \%$; $\mathrm{n}=143)$, the ORR was $15.5 \%$ (95\% CI, 10.1-22.4), with $2 \%$ (95\% CI, 0.4-5.8) of patients achieving a complete response. The median duration of response was 16.3 months. Investigations involving cohorts 2 and 3 of the KEYNOTE-059 trial, which will examine the 
efficacy of first-line pembrolizumab in combination with chemotherapy or as a single agent, are ongoing (ClinicalTrials.gov Identifier: NCT02335411)..$^{145-147}$ Preliminary results suggest that pembrolizumab as a single agent or in combination with CF demonstrates promising antitumor activity and acceptable toxicity as first-line therapy for PD-L1-positive advanced gastric and EGJ cancers. First-line treatment with pembrolizumab in combination with CF will also be investigated in the phase III randomized KEYNOTE590 trial, which is actively recruiting participants with advanced esophageal adenocarcinoma, esophageal SCC, and EGJ adenocarcinoma (ClinicalTrials.gov identifier: NCT03189719). ${ }^{148}$

The recently published KEYNOTE-061 trial directly compared monotherapy with pembrolizumab to paclitaxel in patients with advanced gastric or EGJ cancers that progressed following first-line therapy with combined fluoropyrimidine and platinum-based agents. ${ }^{149}$ In this multicenter international phase III trial, 395 patients with PD-L1-positive tumors were randomized to receive either pembrolizumab $(n=196)$ or standard-dose paclitaxel $(\mathrm{n}=199)$. Median OS was 9.1 months $(95 \% \mathrm{CI}$, 6.2-10.7) with pembrolizumab and 8.3 months (95\% CI, 7.6-9.0) with paclitaxel (HR=0.82, 95\% CI, 0.66-1.03; $P=.0421)$. Median PFS was 1.5 months (95\% CI, 1.4-2.0) and 4.1 months (95\% CI, 3.1-4.2), respectively ( $\mathrm{HR}=1.27$; 95\% CI, 1.03-1.57). Grade 3-5 treatment-related adverse events occurred in $14 \%$ of the patients treated with pembrolizumab compared with $35 \%$ of the patients treated with paclitaxel. Therefore, although pembrolizumab did not significantly improve OS compared with paclitaxel as second-line therapy for advanced PD-L1-positive gastric or EGJ cancer, pembrolizumab had a better safety profile and was better tolerated by patients. Additionally, Doi et $\mathrm{al}^{150}$ recently analyzed preliminary data from the advanced esophageal cancer cohort $(n=23)$ of the KEYNOTE028 trial, a multicohort phase Ib trial of pembrolizumab in patients with PD-L1-positive advanced solid tumors that have failed first-line therapy. In patients with adenocarcinoma of the esophagus or EGJ, the ORR was $40 \%$. Median PFS was 1.8 months (95\% CI, 1.7-2.9) and the 6- and 12 -month PFS rates were $30 \%$ and $22 \%$, respectively. Median OS was 7 months (95\% CI, 4.3-17.7) and the 6and 12 -month OS rates were $60 \%$ and $40 \%$, respectively. Grade 3 immune-mediated adverse events, including decreased appetite and decreased lymphocyte count, occurred in $17 \%$ of patients, but no grade 4 adverse events were reported.

Two of the most recently published KEYNOTE trials (KEYNOTE-180 and KEYNOTE-181) examined the efficacy of pembrolizumab in treating PD-L1-positive esophageal or EGJ tumors defined as having a CPS $\geq 10 .{ }^{37,151}$ This is in contrast to previous studies that have defined PD-L1positive tumors as having a CPS $\geq 1$. In the phase II single-arm KEYNOTE-180 trial, which evaluated pembrolizumab monotherapy in 121 patients with progressive disease after $\geq 2$ prior lines of therapy, the objective response rate was $9.9 \%(95 \% \mathrm{CI}, 5.2 \%-16.7 \%)$ among all patients, $5.2 \%$ (95\% CI, 1.1\%-14.4\%) among patients with adenocarcinoma $(n=58)$, and $13.8 \%(95 \%$ CI, 6.1\%-25.4\%) among patients with PD-L1-positive tumors $(\mathrm{n}=58) .{ }^{151}$ Overall, $12.4 \%$ of patients experience grade 3-5 treatment-related adverse events. These results demonstrated the efficacy and tolerability of pembrolizumab as third-line or subsequent therapy in heavily pretreated esophageal cancers with high PD-L1 expression. The phase III KEYNOTE-181 trial evaluated pembrolizumab versus investigator's choice of chemotherapy (docetaxel, paclitaxel or irinotecan) as secondline therapy in 628 patients with advanced SCC or adenocarcinoma of the esophagus or EGJ. ${ }^{37}$ Patients (401 with SCC and 222 with PD-L1 CPS $\geq 10$ ) were randomized 1:1 to pembrolizumab or chemotherapy and randomization was stratified by histology (SCC vs adenocarcinoma) and region (Asia vs rest of world). Pembrolizumab significantly improved median OS (9.3 vs 6.7 months; $\mathrm{HR}=0.69 ; 95 \% \mathrm{CI}, 0.52-0.93 ; P=.0074$ ) and 12 -month OS rates ( $43 \%$ vs $20 \%$ ) compared with chemotherapy in patients whose tumors had a PD-L1 CPS $\geq 10$. Although the difference in OS was not statistically significant (7.1 vs 7.1 months; $\mathrm{HR}=0.89 ; 95 \% \mathrm{CI}, 0.75-1.05$; $P=.0560$ ), fewer patients ( $18 \%$ vs $41 \%$ ) had grade $3-5$ treatment-related adverse events with pembrolizumab compared with chemotherapy. These data suggest that pembrolizumab may be an effective second-line therapy for patients with advanced esophageal cancer with a PD-L1 CPS $\geq 10$, with a more favorable safety profile than chemotherapy.

\section{Treatment Guidelines}

The management of patients with esophageal and EGJ cancers requires the expertise of several disciplines, including surgical oncology, medical oncology, gastroenterology, radiation oncology, radiology, and pathology. In addition, the presence of nutritional services, social workers, nurses, palliative care specialists, and other supporting disciplines are also desirable. Hence, the panel believes in an infrastructure that encourages multidisciplinary treatment decision-making by members of all disciplines taking care of patients with esophageal and EGJ cancers. The recommendations made by the multidisciplinary team may be considered advisory to the primary group of treating physicians of the patient.

\section{Workup}

Newly diagnosed patients should undergo a complete history and physical examination, complete blood count, comprehensive chemistry profile, and upper GI endoscopy 
with biopsy of the primary tumor (see ESOPH-1, page 856). Histologic evaluation is required for correct diagnosis of SCC or adenocarcinoma. The extent of tumor involvement into the EGJ and cardia should be clearly documented, where applicable. CT scan (with oral and intravenous contrast) of the chest and abdomen should also be performed. Pelvic CT with contrast should be obtained when clinically indicated. EUS and FDGPET/CT evaluation from skull base to midthigh are recommended if metastatic disease is not evident. HER2, MSI-H/dMMR, and PD-L1 testing are recommended at the time of diagnosis if metastatic disease is documented or suspected. Assessment of Siewert tumor type should be included as part of the initial workup in all patients with EGJ adenocarcinoma. ${ }^{16,17}$ The guidelines also recommend screening for family history of esophageal or EGJ cancers. Referral to a cancer genetics professional is recommended for those with a family history or a known high-risk syndrome associated with esophageal and EGJ cancers.

Initial workup enables patients to be classified into 2 clinical stage groups:

- Locoregional cancer (stage I-III)

- Metastatic cancer (stage IV)

\section{Additional Evaluation}

Additional evaluations are warranted to assess a patient's medical condition, ability to tolerate major surgery, and the feasibility of resection (see ESOPH-11, page 857). These evaluations may include pulmonary function studies, cardiac testing, and nutritional assessment. An enteric feeding tube should be considered in surgical candidates for preoperative nutritional support. A jejunostomy tube is preferred, but a percutaneous gastrostomy tube may be considered for patients with cervical esophageal tumors receiving definitive chemoradiation or for patients with marginally resectable disease. Multidisciplinary expertise is recommended before placement of a percutaneous gastrostomy tube. The approach, timing, and location of the feeding tube should be discussed with the surgeon before placement. Laparoscopy is optional for EGJ adenocarcinoma if there is no evidence of metastatic disease. Colonoscopy may be warranted if colon interposition is planned as part of the surgical procedure. A superior mesenteric artery angiogram should be considered only in select patients when colon interposition is planned.

Additional evaluation enables patients with locoregional cancer to be further classified into the following groups:

- Medically fit for surgery

- Nonsurgical candidates (medically unable to tolerate major surgery or medically fit patients who decline surgery)

\section{Primary Treatment of \\ Locoregional Adenocarcinoma}

\section{Medically Fit Patients}

Esophagectomy is indicated for patients with cTlbcT2, N0 low-risk lesions $(<2 \mathrm{~cm}$ in diameter and welldifferentiated; see ESOPH-13, page 858). Endoscopic resection is appropriate for many Tla lesions, but it is important to understand that staging of early esophageal cancer (T1 and even some T2 disease) is challenging via existing imaging techniques, including EUS. Thus, if there is a question of depth of invasion for early esophageal malignancy, endoscopic resection provides both diagnostic and potentially curative therapy for Tla and some early Tlb disease. Primary treatment options for patients with cT1b-cT2, N+ or cT3-cT4a, any N tumors include preoperative chemoradiation (category 1; preferred), ${ }^{38}$ definitive chemoradiation (only for patients who decline surgery), ${ }^{57,58,85}$ perioperative chemotherapy, ${ }^{39,40}$ and preoperative chemotherapy ${ }^{53}$ Definitive chemoradiation is the primary treatment option for patients with cT4b (unresectable) tumors and occasionally can facilitate surgical resection in select patients. ${ }^{152}$ Chemotherapy alone can be considered for these patients in the setting of invasion of the trachea, great vessels, or heart.

\section{Non-Surgical Candidates}

Definitive chemoradiation is recommended for nonsurgical candidates with cT1b-cT4b, any N tumors who are able to tolerate chemoradiation (see ESOPH-17, page 862). Palliative RT or palliative best supportive care are the appropriate options for nonsurgical candidates who are unable to tolerate chemoradiation.

Response Assessment and Additional Management Additional management options are based on the assessment of response to primary treatment. FDG-PET/ CT scans are useful for the evaluation of patients after chemoradiation and before surgery for the detection of distant metastases. ${ }^{153,154}$ Therefore, assessment with FDG-PET/CT (preferred) or FDG-PET scan should be done $\geq 5$ to 8 weeks after the completion of preoperative therapy (see ESOPH-14, page 859). Chest/abdominal CT scan with contrast is recommended, but is not required if FDG-PET/CT was done. Pelvic CT with contrast can be considered for distal lesions, if clinically indicated. Upper GI endoscopy and biopsy is recommended following definitive chemoradiation, but it is optional after preoperative chemoradiation if surgery is planned.

Esophagectomy (preferred) or surveillance (category 2B) are recommended for patients with no evidence of disease after preoperative chemoradiation. Esophagectomy is also preferred for those with persistent local disease after preoperative chemoradiation. Patients with 
no evidence of disease following definitive chemoradiation should be managed with surveillance, while esophagectomy is recommended for those with persistent local disease. Alternatively, patients with persistent local disease or unresectable/metastatic disease after either preoperative or definitive chemoradiation can be managed with palliative or best supportive care.

\section{Postoperative Management}

Postoperative management is based on surgical margins, nodal status, histology, and previous treatment. The components of postoperative management have not been established in randomized trials for patients with esophageal cancer. Available evidence for the use of postoperative chemoradiation and postoperative chemotherapy comes from prospective randomized trials involving patients with gastric cancer. ${ }^{54-56}$

\section{Patients Who Have Not Received Preoperative Chemoradiation or Chemotherapy}

Surveillance is recommended for patients with R0 resection and negative nodal status (see ESOPH-15, page 860). Chemoradiation is an alternative option following R0 resection for patients with pT3-pT4a tumors or select patients with pT2 tumors in the lower esophagus or EGJ and high-risk features (category 2B). ${ }^{54,55}$ High-risk features include poorly differentiated or higher grade cancer, lymphovascular invasion, perineural invasion, or age $<50$ years. For patients with R0 resection and positive nodal status, chemoradiation ${ }^{54,55}$ or chemotherapy is recommended. Patients with $\mathrm{R} 1$ resection should receive chemoradiation while those with $\mathrm{R} 2$ resection can receive either chemoradiation or palliative management.

\section{Patients Who Have Received Preoperative Chemoradiation or Chemotherapy}

Observation until disease progression is recommended for all patients with $\mathrm{R} 0$ resection who had received preoperative therapy, irrespective of their nodal status (see ESOPH-16, page 861). If received perioperatively, postoperative chemotherapy is a category 1 recommendation for patients with completely resected, node-negative, or node-positive disease. ${ }^{39,40}$ Based on current data, adjuvant chemoradiation is not recommended for patients with node-positive disease after R0 resection. Patients with R1 or $\mathrm{R} 2$ resection should be treated with chemoradiation, if not received preoperatively. Alternatively, patients with $\mathrm{R} 1$ resection can be observed until disease progression or considered for reresection. Palliative management is an alternative option for patients with $\mathrm{R} 2$ resection.

\section{Follow-up/Surveillance}

All patients should be followed up systematically. However, surveillance strategies after successful local therapy of esophageal and EGJ cancers remain controversial, with no high-level evidence to guide development of algorithms that balance benefits and risks (including cost) within this cohort. The stage-specific surveillance strategies provided in this guideline are based on currently available evidence from retrospective studies ${ }^{68,155-159}$ and expert consensus. In general, follow-up for asymptomatic patients should include a complete history and physical examination every 3 to 6 months for the first 2 years, every 6 to 12 months for years 3 to 5 , and then annually thereafter (see ESOPH-18, page 863). Complete blood count, chemistry profile, upper GI endoscopy with biopsy, and imaging studies should be performed as clinically indicated. In addition, some patients may require dilatation of an anastomotic or a chemoradiationinduced stricture. Nutritional assessment and counseling are also recommended.

\section{Unresectable, Locally Advanced, Recurrent, or Metastatic Disease}

When locoregional recurrence develops after prior chemoradiation therapy, the clinician should determine whether the patient is medically fit for surgery and if the recurrence is resectable. If both criteria are met, esophagectomy remains an option (see ESOPH18, page 863). Palliative management, which includes concurrent chemoradiation (preferred), surgery, chemotherapy, and palliative or best supportive care, is recommended for patients who develop a locoregional recurrence after prior esophagectomy. Those who are medically unable to tolerate major surgery and those who develop an unresectable or metastatic recurrence should also receive palliative management. If not done previously, HER2, MSI-H/dMMR, and PD-L1 testing should be performed in patients with suspected metastatic disease.

Palliative management and best supportive care are always indicated for patients with unresectable locally advanced, recurrent, or metastatic disease (see ESOPH-19, page 864). The decision to offer palliative/best supportive care alone or with systemic therapy depends on the patient's performance status. The Eastern Cooperative Oncology Group Performance Status Scale (ECOG PS) and the Karnofsky Performance Status Scale (KPS) are commonly used to assess the performance status of patients with cancer. ${ }^{160-162}$ Patients with a KPS score $<60 \%$ or an ECOG PS score $\geq 3$ should be offered palliative or best supportive care only. Systemic therapy can be offered in addition to palliative or best supportive care for patients with better performance status (KPS score $\geq 60 \%$ or ECOG PS score $\leq 2$ ). Dysphagia should also be assessed since it has a significant impact on quality of life and is often amenable to palliation regardless of performance status. Dysphagia is most 
often palliated via endoscopic stenting, but patients should be counseled on reflux and chest pain associated with stent placement. Alternatively, external beam radiation therapy or brachytherapy, among other potential modalities, can be used.

The survival benefit of systemic therapy compared with palliative or best supportive care alone has been shown in small cohorts of patients with esophageal or EGJ adenocarcinoma included in gastric adenocarcinoma trials. ${ }^{88,89}$ A recent Cochrane database systematic review analyzed 5 randomized controlled trials (involving 750 patients) comparing palliative chemotherapy and/or targeted therapy to best supportive care alone in patients with advanced esophageal or EGJ cancer..$^{90}$ The analysis demonstrated a benefit in OS for patients receiving palliative therapy (chemotherapy or targeted therapy) compared with those receiving best supportive care alone $(\mathrm{HR}=0.81 ; 95 \% \mathrm{CI}, 0.71-0.92)$. The only individual agent found by more than one study to improve both OS and PFS was ramucirumab. Although the addition of palliative chemotherapy or targeted therapy increased the frequency of grade $\geq 3$ adverse events, treatment-related deaths did not increase. Importantly, patient-reported quality of life often improved with the addition of palliative systemic therapy to best supportive care. Therefore, the addition of systemic therapy to best supportive care can improve the quality of life and may prolong survival in patients with advanced esophageal or EGJ cancers.

\section{Summary}

The NCCN Guidelines for Esophageal and Esophagogastric Junction Cancers provide an evidence- and consensusbased treatment approach for the management of patients with esophageal and EGJ cancers. Multidisciplinary team management is essential for all patients with esophageal and EGJ cancers. Combined modality therapy, especially preoperative chemoradiation, is recommended for locally advanced disease. Best supportive care is an integral part of treatment, especially in patients with locally advanced or metastatic disease. Targeted therapies have produced encouraging results in the treatment of patients with advanced esophageal and EGJ cancers. Trastuzumab plus chemotherapy is recommended as first-line therapy for patients with HER2positive metastatic adenocarcinoma. Ramucirumab, as a single agent or in combination with paclitaxel, and pembrolizumab (for MSI-H/dMMR tumors) are included as options for second-line or subsequent therapy for patients with metastatic disease. Pembrolizumab has also been included as a second-line therapy option for esophageal cancers with PD-L1 expression levels by CPS of $\geq 10$ and as a third-line or subsequent therapy option for esophageal and EGJ adenocarcinoma with PD-L1 expression levels by CPS of $\geq 1$. The panel encourages patients with esophageal and EGJ cancers to participate in well-designed clinical trials investigating novel therapeutic strategies to enable further advances.

\section{References}

1. Torre LA, Siegel RL, Ward EM, et al. Global cancer incidence and mortality rates and trends-an update. Cancer Epidemiol Biomarkers Prev 2016:25:16-27.

2. World Health Organization. International Agency for Research on Cancer. GLOBOCAN 2018: oesophagus cancer fact sheet. 2018. Available at: http://gco.iarc.fr/today/data/factsheets/cancers/ 6-Oesophagus-fact-sheet.pdf. Accessed January 22, 2019

3. Torre LA, Bray F, Siegel RL, et al. Global cancer statistics, 2012. CA Cancer J Clin 2015;65:87-108.

4. Siegel RL, Miller KD, Jemal A. Cancer statistics, 2019. CA Cancer J Clin 2019;69:7-34.

5. World Health Organization. International Agency for Research on Cancer. GLOBOCAN 2018: United States of America cancer fact sheet. 2018. Available at: http://gco.iarc.fr/today/data/factsheets/populations/ 840-united-states-of-america-fact-sheets.pdf. Accessed January 22, 2019.

6. Siewert JR, Ott K. Are squamous and adenocarcinomas of the esophagus the same disease? Semin Radiat Oncol 2007;17:38-44.

7. Amin MB, Edge SB, Greene FL, editors. AJCC cancer staging manual, 8th Ed. Springer, New York, NY. 2017

8. Turati F, Tramacere I, La Vecchia C, et al. A meta-analysis of body mass index and esophageal and gastric cardia adenocarcinoma. Ann Oncol 2013;24:609-617.

9. Ryan AM, Duong $M$, Healy $L$, et al. Obesity, metabolic syndrome and esophageal adenocarcinoma: epidemiology, etiology and new targets. Cancer Epidemiol 2011;35:309-319

10. Engel LS, Chow W-H, Vaughan TL, et al. Population attributable risks of esophageal and gastric cancers. J Natl Cancer Inst 2003;95:1404-1413.

11. Lagergren J, Bergström R, Lindgren A, et al. The role of tobacco, snuff and alcohol use in the aetiology of cancer of the oesophagus and gastric cardia. Int J Cancer 2000;85:340-346.
12. Freedman ND, Abnet CC, Leitzmann MF, et al. A prospective study of tobacco, alcohol, and the risk of esophageal and gastric cancer subtypes. Am J Epidemiol 2007;165:1424-1433.

13. Rice TW, Apperson-Hansen C, DiPaola LM, et al. Worldwide Esophageal Cancer Collaboration: clinical staging data. Dis Esophagus 2016;29: 707-714

14. Rice TW, Gress DM, Patil DT, et al. Cancer of the esophagus and esophagogastric junction-major changes in the American Joint Committee on Cancer eighth edition cancer staging manual. CA Cancer J Clin 2017;67:304-317.

15. Kim TJ, Kim HY, Lee KW, et al. Multimodality assessment of esophageal cancer: preoperative staging and monitoring of response to therapy. Radiographics 2009;29:403-421.

16. Siewert JR, Stein H. Carcinoma of the cardia: carcinoma of the gastroesophageal junction - classification, pathology, and extent of resection. Dis Esophagus 1996;9:173-182.

17. Rüdiger Siewert J, Feith $M$, Werner $M$, et al. Adenocarcinoma of the esophagogastric junction: results of surgical therapy based on anatomical/topographic classification in 1,002 consecutive patients. Ann Surg 2000;232:353-361.

18. Hechtman JF, Polydorides AD. HER2/neu gene amplification and protein overexpression in gastric and gastroesophageal junction adenocarcinoma: a review of histopathology, diagnostic testing, and clinical implications. Arch Pathol Lab Med 2012;136:691-697.

19. Moelans CB, van Diest PJ, Milne AN, et al. Her-2/neu testing and therapy in gastroesophageal adenocarcinoma. Pathol Res Int 2010;2011: 674182.

20. Kato S, Okamura R, Baumgartner JM, et al. Analysis of circulating tumor DNA and clinical correlates in patients with esophageal, gastroesophageal junction, and gastric adenocarcinoma. Clin Cancer Res 2018; 24:6248-6256. 
21. Dreilich M, Wanders A, Brattström D, et al. HER-2 overexpression (3+) in patients with squamous cell esophageal carcinoma correlates with poorer survival. Dis Esophagus 2006;19:224-231.

22. Reichelt $U$, Duesedau $P$, Tsourlakis MC, et al. Frequent homogeneous HER-2 amplification in primary and metastatic adenocarcinoma of the esophagus. Mod Pathol 2007;20:120-129.

23. Schoppmann SF, Jesch B, Friedrich J, et al. Expression of Her-2 in carcinomas of the esophagus. Am J Surg Pathol 2010;34:1868-1873.

24. Gravalos C, Jimeno A. HER2 in gastric cancer: a new prognostic factor and a novel therapeutic target. Ann Oncol 2008;19:1523-1529.

25. Tanner M, Hollmén M, Junttila TT, et al. Amplification of HER-2 in gastric carcinoma: association with Topoisomerase Ilalpha gene amplification, intestinal type, poor prognosis and sensitivity to trastuzumab. Ann Oncol 2005; 16:273-278.

26. Bang YJ, Van Cutsem E, Feyereislova A, et al. Trastuzumab in combination with chemotherapy versus chemotherapy alone for treatment of HER2-positive advanced gastric or gastro-oesophageal junction cancer (ToGA): a phase 3, open-label, randomised controlled trial. Lancet 2010;376:687-697.

27. Bang $\mathrm{Y}, \mathrm{Chung} \mathrm{H}, \mathrm{Xu} \mathrm{J}$, et al. Pathological features of advanced gastric cancer (GC): relationship to human epidermal growth factor receptor 2 (HER2) positivity in the global screening programme of the ToGA trial [abstract]. J Clin Oncol 2009;27: Abstract 4556.

28. Bartley AN, Washington MK, Colasacco C, et al. HER2 testing and clinical decision making in gastroesophageal adenocarcinoma: guideline from the College of American Pathologists, American Society for Clinical Pathology, and the American Society of Clinical Oncology. J Clin Oncol 2017;35:446-464.

29. Hofmann M, Stoss $O$, Shi D, et al. Assessment of a HER2 scoring system for gastric cancer: results from a validation study. Histopathology 2008; 52:797-805.

30. Rüschoff J, Dietel M, Baretton $\mathrm{G}$, et al. HER2 diagnostics in gastric cancer-guideline validation and development of standardized immunohistochemical testing. Virchows Arch 2010;457:299-307.

31. U.S. Food and Drug Administration. FDA grants accelerated approval to pembrolizumab for first tissue/site agnostic indication. 2017. Available at: https://www.fda.gov/drugs/informationondrugs/approveddrugs/ ucm560040.htm. Accessed February 21, 2019.

32. Le DT, Durham JN, Smith KN, et al. Mismatch repair deficiency predicts response of solid tumors to PD-1 blockade. Science 2017;357:409-413.

33. Murphy KM, Zhang $S$, Geiger $T$, et al. Comparison of the microsatellite instability analysis system and the Bethesda panel for the determination of microsatellite instability in colorectal cancers. J Mol Diagn 2006;8: 305-311.

34. Bartley AN, Fitzgibbons PL, Broaddus RR, et al. Template for reporting results of DNA mismatch repair testing in patients being considered for checkpoint inhibitor immunotherapy. Northfield, Illinois: College of American Pathologists; 2018.

35. U.S. Food and Drug Administration. FDA grants accelerated approval to pembrolizumab for advanced gastric cancer. 2017. Available at: https://www.fda.gov/Drugs/InformationOnDrugs/ApprovedDrugs/ ucm577093.htm. Accessed February 21, 2019.

36. Fuchs CS, Doi T, Jang RW, et al. Safety and efficacy of pembrolizumab monotherapy in patients with previously treated advanced gastric and gastroesophageal junction cancer: phase 2 clinical KEYNOTE-059 trial. JAMA Oncol 2018;4:e180013.

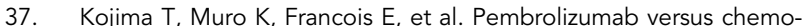
therapy as second-line therapy for advanced esophageal cancer: phase III KEYNOTE-181 study. J Clin Oncol 2019;37(4_suppl):2.

38. van Hagen $\mathrm{P}$, Hulshof MC, van Lanschot JJ, et al. Preoperative chemoradiotherapy for esophageal or junctional cancer. N Engl J Med 2012;366:2074-2084.

39. Ychou $M$, Boige $V$, Pignon J-P, et al. Perioperative chemotherapy compared with surgery alone for resectable gastroesophageal adenocarcinoma: an FNCLCC and FFCD multicenter phase III trial. J Clin Oncol 2011;29:1715-1721.

40. Al-Batran SE, Hofheinz RD, Pauligk C, et al. Histopathological regression after neoadjuvant docetaxel, oxaliplatin, fluorouracil, and leucovorin versus epirubicin, cisplatin, and fluorouracil or capecitabine in patients with resectable gastric or gastro-oesophageal junction adenocarcinoma (FLOT4-AIO): results from the phase 2 part of a multicentre, open-label, randomised phase 2/3 trial. Lancet Oncol 2016;17:1697-1708.

41. Steyerberg EW, Neville BA, Koppert LB, et al. Surgical mortality in patients with esophageal cancer: development and validation of a simple risk score. J Clin Oncol 2006:24:4277-4284.
42. Tirumani $\mathrm{H}$, Rosenthal $\mathrm{MH}$, Tirumani $\mathrm{SH}$, et al. Esophageal carcinoma: current concepts in the role of imaging in staging and management. Can Assoc Radiol J 2015;66:130-139.

43. Rusch VW. Are cancers of the esophagus, gastroesophageal junction, and cardia one disease, two, or several? Semin Oncol 2004; 31:444-449.

44. Siewert JR, Stein HJ, Feith M. Adenocarcinoma of the esophago-gastric junction. Scand J Surg 2006;95:260-269.

45. de Graaf GW, Ayantunde AA, Parsons SL, et al. The role of staging laparoscopy in oesophagogastric cancers. Eur J Surg Oncol 2007;33: 988-992.

46. Nath J, Moorthy K, Taniere P, et al. Peritoneal lavage cytology in patients with oesophagogastric adenocarcinoma. Br J Surg 2008; 95:721-726

47. Peyre CG, Hagen JA, DeMeester SR, et al. Predicting systemic disease in patients with esophageal cancer after esophagectomy: a multinational study on the significance of the number of involved lymph nodes. Ann Surg 2008;248:979-985

48. Rizk NP, Ishwaran H, Rice TW, et al. Optimum lymphadenectomy for esophageal cancer. Ann Surg 2010;251:46-50.

49. Groth SS, Virnig BA, Whitson BA, et al. Determination of the minimum number of lymph nodes to examine to maximize survival in patients with esophageal carcinoma: data from the Surveillance Epidemiology and End Results database. J Thorac Cardiovasc Surg 2010;139:612-620.

50. Coccolini F, Nardi M, Montori G, et al. Neoadjuvant chemotherapy in advanced gastric and esophago-gastric cancer. Meta-analysis of randomized trials. Int J Surg 2018;51:120-127.

51. Al-Batran SE, Lorenzen S. Management of locally advanced gastroesophageal cancer: still a multidisciplinary global challenge? Hematol Oncol Clin North Am 2017:31:441-452.

52. Kleinberg L, Forastiere AA. Chemoradiation in the management of esophageal cancer. J Clin Oncol 2007;25:4110-4117.

53. Alderson D, Cunningham D, Nankivell $M$, et al. Neoadjuvant cisplatin and fluorouracil versus epirubicin, cisplatin, and capecitabine followed by resection in patients with oesophageal adenocarcinoma (UK MRC OE05): an open-label, randomised phase 3 trial. Lancet Oncol 2017; 18:1249-1260.

54. Smalley SR, Benedetti JK, Haller DG, et al. Updated analysis of SWOG directed intergroup study 0116: a phase III trial of adjuvant radiochemotherapy versus observation after curative gastric cancer resection. J Clin Oncol 2012;30:2327-2333.

55. Macdonald JS, Smalley SR, Benedetti J, et al. Chemoradiotherapy after surgery compared with surgery alone for adenocarcinoma of the stomach or gastroesophageal junction. N Engl J Med 2001;345: 725-730.

56. Noh SH, Park SR, Yang HK, et al.Adjuvant capecitabine plus oxaliplatin for gastric cancer after D2 gastrectomy (CLASSIC): 5-yea follow-up of an open-label, randomised phase 3 trial. Lancet Oncol 2014;15:1389-1396.

57. Conroy T, Galais M-P, Raoul J-L, et al. Definitive chemoradiotherapy with FOLFOX versus fluorouracil and cisplatin in patients with oesophageal cancer (PRODIGE5/ACCORD17): final results of a randomised, phase 2/3 trial. Lancet Oncol 2014;15:305-314.

58. Minsky BD, Pajak TF, Ginsberg RJ, et al. INT 0123 (Radiation Therapy Oncology Group 94-05) phase III trial of combined-modality therapy for esophageal cancer: high-dose versus standard-dose radiation therapy. $\mathrm{J}$ Clin Oncol 2002;20:1167-1174.

59. Li QQ, Liu MZ, Hu YH, et al. Definitive concomitant chemoradiotherapy with docetaxel and cisplatin in squamous esophageal carcinoma. Dis Esophagus 2010;23:253-259.

60. Day FL, Leong T, Ngan S, et al. Phase I trial of docetaxel, cisplatin and concurrent radical radiotherapy in locally advanced oesophageal cancer. Br J Cancer 2011;104:265-271.

61. lyer R, Wilkinson $N$, Demmy $T$, et al. Controversies in the multimodality management of locally advanced esophageal cancer: evidence-based review of surgery alone and combined-modality therapy. Ann Surg Oncol 2004;11:665-673.

62. Urschel JD, Vasan H. A meta-analysis of randomized controlled trials that compared neoadjuvant chemoradiation and surgery to surgery alone for resectable esophageal cancer. Am J Surg 2003;185:538-543.

63. Fiorica F, Di Bona D, Schepis F, et al. Preoperative chemoradiotherapy for oesophageal cancer: a systematic review and meta-analysis. Gut 2004:53:925-930. 
64. Ma HF, Lv GX, Cai ZF, et al. Comparison of the prognosis of neoadjuvant chemoradiotherapy treatment with surgery alone in esophageal carcinoma: a meta-analysis. OncoTargets Ther 2018;11:3441-3447.

65. Sjoquist KM, Burmeister BH, Smithers BM, et al. Survival after neoadjuvant chemotherapy or chemoradiotherapy for resectable oesophageal carcinoma: an updated meta-analysis. Lancet Oncol 2011;12:681-692.

66. Swisher SG, Hofstetter W, Komaki R, et al. Improved long-term outcome with chemoradiotherapy strategies in esophageal cancer. Ann Thorac Surg 2010;90:892-898[discussion 898-899].

67. Zhao X, Ren Y, Hu Y, et al. Neoadjuvant chemotherapy versus neoadjuvant chemoradiotherapy for cancer of the esophagus or the gastroesophageal junction: A meta-analysis based on clinical trials. PLoS One 2018;13:e0202185.

68. Oppedijk V, van der Gaast A, van Lanschot JJ, et al. Patterns of recurrence after surgery alone versus preoperative chemoradiotherapy and surgery in the CROSS trials. J Clin Oncol 2014;32:385-391.

69. Noordman BJ, Verdam MGE, Lagarde SM, et al. Effect of neoadjuvant chemoradiotherapy on health-related quality of life in esophageal or junctional cancer: results from the randomized CROSS trial. J Clin Oncol 2018;36:268-275

70. Shapiro J, van Lanschot JJB, Hulshof MCCM, et al. Neoadjuvant chemoradiotherapy plus surgery versus surgery alone for oesophageal or junctional cancer (CROSS): long-term results of a randomised controlled trial. Lancet Oncol 2015;16:1090-1098.

71. Leichman LP, Goldman BH, Bohanes PO, et al. S0356: a phase II clinical and prospective molecular trial with oxaliplatin, fluorouracil, and external-beam radiation therapy before surgery for patients with esophageal adenocarcinoma. J Clin Oncol 2011;29:4555-4560.

72. Khushalani NI, Leichman CG, Proulx G, et al. Oxaliplatin in combination with protracted-infusion fluorouracil and radiation: report of a clinical trial for patients with esophageal cancer. J Clin Oncol 2002; 20:2844-2850

73. Tepper J, Krasna MJ, Niedzwiecki D, et al. Phase III trial of trimodality therapy with cisplatin, fluorouracil, radiotherapy, and surgery compared with surgery alone for esophageal cancer: CALGB 9781. J Clin Oncol 2008;26:1086-1092.

74. Bedenne L, Michel P, Bouché O, et al. Chemoradiation followed by surgery compared with chemoradiation alone in squamous cancer of the esophagus: FFCD 9102. J Clin Oncol 2007;25:1160-1168.

75. Sharma R, Yang GY, Nava HR, et al. A single institution experience with neoadjuvant chemoradiation (CRT) with irinotecan (I) and cisplatin (C) in locally advanced esophageal carcinoma (LAEC) [abstract]. J Clin Oncol 2009:27:e15619.

76. Ajani JA, Winter K, Okawara GS, et al. Phase II trial of preoperative chemoradiation in patients with localized gastric adenocarcinoma (RTOG 9904): quality of combined modality therapy and pathologic response. J Clin Oncol 2006;24:3953-3958.

77. Cunningham D, Allum WH, Stenning SP, et al. Perioperative chemotherapy versus surgery alone for resectable gastroesophageal cancer. N Engl J Med 2006;355:11-20.

78. Al-Batran S-E, Homann N, Pauligk C, et al. Perioperative chemotherapy with fluorouracil plus leucovorin, oxaliplatin, and docetaxel versus fluorouracil or capecitabine plus cisplatin and epirubicin for locally advanced, resectable gastric or gastro-oesophageal junction adenocarcinoma (FLOT4): a randomised, phase 2/3 trial. Lancet 2019. 393:1948-1957.

79. Kofoed SC, Muhic A, Baeksgaard L, et al. Survival after adjuvant chemoradiotherapy or surgery alone in resectable adenocarcinoma at the gastro-esophageal junction. Scand J Surg 2012;101:26-31.

80. Medical Research Council Oesophageal Cancer Working Group. Surgical resection with or without preoperative chemotherapy in oesophageal cancer: a randomised controlled trial. Lancet 2002; 359:1727-1733.

81. Allum WH, Stenning SP, Bancewicz J, et al. Long-term results of a randomized trial of surgery with or without preoperative chemotherapy in esophageal cancer. J Clin Oncol 2009;27:5062-5067.

82. Boonstra JJ, Kok TC, Wijnhoven BP, et al. Chemotherapy followed by surgery versus surgery alone in patients with resectable oesophageal squamous cell carcinoma: long-term results of a randomized controlled trial. BMC Cancer 2011;11:181.

83. Ruppert BN, Watkins JM, Shirai K, et al. Cisplatin/irinotecan versus carboplatin/paclitaxel as definitive chemoradiotherapy for locoregionally advanced esophageal cancer. Am J Clin Oncol 2010;33:346-352.
84. Herskovic A, Martz K, al-Sarraf M, et al. Combined chemotherapy and radiotherapy compared with radiotherapy alone in patients with cancer of the esophagus. N Engl J Med 1992;326:1593-1598.

85. Cooper JS, Guo MD, Herskovic A, et al. Chemoradiotherapy of locally advanced esophageal cancer: long-term follow-up of a prospective randomized trial (RTOG 85-01). JAMA 1999;281:1623-1627.

86. Bascoul-Mollevi C, Gourgou S, Galais MP, et al. Health-related quality of life results from the PRODIGE 5/ACCORD 17 randomised trial of FOLFOX versus fluorouracil-cisplatin regimen in oesophageal cancer. Eur J Cancer 2017;84:239-249.

87. Bang YJ, Kim YW, Yang HK, et al. Adjuvant capecitabine and oxaliplatin for gastric cancer after D2 gastrectomy (CLASSIC): a phase 3 open-label, randomised controlled trial. Lancet 2012;379:315-321.

88. Ford HE, Marshall A, Bridgewater JA, et al. Docetaxel versus active symptom control for refractory oesophagogastric adenocarcinoma (COUGAR-02): an open-label, phase 3 randomised controlled trial. Lancet Oncol 2014;15:78-86.

89. Thuss-Patience PC, Kretzschmar A, Bichev D, et al. Survival advantage for irinotecan versus best supportive care as second-line chemotherapy in gastric cancer--a randomised phase III study of the Arbeitsgemeinschaft Internistische Onkologie (AIO). Eur J Cancer 2011;47:2306-2314.

90. Janmaat VT, Steyerberg EW, van der Gaast A, et al. Palliative chemotherapy and targeted therapies for esophageal and gastroesophageal junction cancer. Cochrane Database Syst Rev 2017;11:CD004063.

91. Al-Batran S-E, Hartmann JT, Probst $\mathrm{S}$, et al. Phase III trial in metastatic gastroesophageal adenocarcinoma with fluorouracil, leucovorin plus either oxaliplatin or cisplatin: a study of the Arbeitsgemeinschaft Internistische Onkologie. J Clin Oncol 2008;26:1435-1442.

92. Enzinger PC, Burtness BA, Niedzwiecki D, et al. CALGB 80403 (Alliance)/ E1206: a randomized phase II study of three chemotherapy regimens plus cetuximab in metastatic esophageal and gastroesophageal junction cancers. J Clin Oncol 2016;34:2736-2742.

93. Kim GM, Jeung HC, Rha SY, et al. A randomized phase II trial of S-1oxaliplatin versus capecitabine-oxaliplatin in advanced gastric cancer. Eur J Cancer 2012;48:518-526.

94. Lorenzen S, Schuster T, Porschen R, et al. Cetuximab plus cisplatin5-fluorouracil versus cisplatin-5-fluorouracil alone in first-line metastatic squamous cell carcinoma of the esophagus: a randomized phase II study of the Arbeitsgemeinschaft Internistische Onkologie. Ann Oncol 2009; 20:1667-1673.

95. Bouché O, Raoul JL, Bonnetain F, et al. Randomized multicenter phase II trial of a biweekly regimen of fluorouracil and leucovorin (LV5FU2), LV5FU2 plus cisplatin, or LV5FU2 plus irinotecan in patients with previously untreated metastatic gastric cancer: a Federation Francophone de Cancerologie Digestive Group Study--FFCD 9803. J Clin Oncol 2004;22:4319-4328.

96. Kang YK, Kang WK, Shin DB, et al. Capecitabine/cisplatin versus 5-fluorouracil/cisplatin as first-line therapy in patients with advanced gastric cancer: a randomised phase III noninferiority trial. Ann Oncol 2009;20:666-673.

97. Park YH, Lee JL, Ryoo BY, et al. Capecitabine in combination with oxaliplatin (XELOX) as a first-line therapy for advanced gastric cancer. Cancer Chemother Pharmacol 2008;61:623-629.

98. Luo HY, Xu RH, Wang F, et al. Phase II trial of XELOX as first-line treatment for patients with advanced gastric cancer. Chemotherapy 2010;56: 94-100.

99. Okines AFC, Norman AR, McCloud P, et al. Meta-analysis of the REAL-2 and ML17032 trials: evaluating capecitabine-based combination chemotherapy and infused 5 -fluorouracil-based combination chemotherapy for the treatment of advanced oesophago-gastric cancer. Ann Oncol 2009:20:1529-1534.

100. Ajani JA, Fodor MB, Tjulandin SA, et al. Phase II multi-institutional randomized trial of docetaxel plus cisplatin with or without fluorouracil in patients with untreated, advanced gastric, or gastroesophageal adenocarcinoma. J Clin Oncol 2005;23:5660-5667.

101. Van Cutsem E, Moiseyenko VM, Tjulandin S, et al. Phase III study of docetaxel and cisplatin plus fluorouracil compared with cisplatin and fluorouracil as first-line therapy for advanced gastric cancer: a report of the V325 Study Group. J Clin Oncol 2006;24:4991-4997.

102. Van Cutsem E, Boni C, Tabernero J, et al. Docetaxel plus oxaliplatin with or without fluorouracil or capecitabine in metastatic or locally recurrent gastric cancer: a randomized phase II study. Ann Oncol 2015;26: 149-156.

103. Shah MA, Janjigian $Y Y$, Stoller $R$, et al. Randomized multicenter phase II study of modified docetaxel, cisplatin, and fluorouracil (DCF) versus DCF 
plus growth factor support in patients with metastatic gastric adenocarcinoma: a study of the US Gastric Cancer Consortium. J Clin Oncol 2015;33:3874-3879

104. Blum Murphy MA, Qiao W, Mewada N, et al. A phase I/II study of docetaxel, oxaliplatin, and fluorouracil (D-FOX) chemotherapy in patients with untreated locally unresectable or metastatic adenocarcinoma of the stomach and gastroesophageal junction. Am J Clin Oncol 2018; 41:321-325.

105. Elsaid AA, Elkerm Y. Final results of a randomized phase III trial of docetaxel, carboplatin and 5FU versus epirubicin, cisplatin and 5FU for locally advanced gastric cancer. J Clin Oncol 2005;23(Suppl 16):4014.

106. Ilson $\mathrm{DH}$. Phase II trial of weekly irinotecan/cisplatin in advanced esophageal cancer. Oncology (Williston Park) 2004; 18(14, Suppl 14) 22-25.

107. Dank M, Zaluski J, Barone C, et al. Randomized phase III study comparing irinotecan combined with 5-fluorouracil and folinic acid to cisplatin combined with 5-fluorouracil in chemotherapy naive patients with advanced adenocarcinoma of the stomach or esophagogastric junction. Ann Oncol 2008;19:1450-1457.

108. Wolff K, Wein A, Reulbach U, et al. Weekly high-dose 5-fluorouracil as a 24-h infusion and sodium folinic acid (AIO regimen) plus irinotecan in patients with locally advanced nonresectable and metastatic adenocarcinoma or squamous cell carcinoma of the oesophagus: a phase II trial. Anticancer Drugs 2009;20:165-173.

109. Burtness B, Gibson M, Egleston B, et al. Phase II trial of docetaxelirinotecan combination in advanced esophageal cancer. Ann Oncol 2009;20:1242-1248.

110. Lustberg MB, Bekaii-Saab T, Young D, et al. Phase II randomized study of two regimens of sequentially administered mitomycin $C$ and irinotecan in patients with unresectable esophageal and gastroesophageal adenocarcinoma. J Thorac Oncol 2010;5:713-718.

111. Moehler M, Kanzler S, Geissler M, et al. A randomized multicenter phase II study comparing capecitabine with irinotecan or cisplatin in metastatic adenocarcinoma of the stomach or esophagogastric junction. Ann Oncol 2010;21:71-77.

112. Guimbaud $R$, Louvet $C$, Ries $P$, et al. Prospective, randomized, multicenter, phase III study of fluorouracil, leucovorin, and irinotecan versus epirubicin, cisplatin, and capecitabine in advanced gastric adenocarcinoma: a French intergroup (Fédération Francophone de Cancérologie Digestive, Fédération Nationale des Centres de Lutte Contre le Cancer, and Groupe Coopérateur Multidisciplinaire en Oncologie) study. J Clin Oncol 2014;32:3520-3526.

113. Ilson DH, Forastiere A, Arquette $\mathrm{M}$, et al. A phase II trial of paclitaxel and cisplatin in patients with advanced carcinoma of the esophagus. Cancer J 2000;6:316-323.

114. Petrasch S, Welt A, Reinacher A, et al. Chemotherapy with cisplatin and paclitaxel in patients with locally advanced, recurrent or metastatic oesophageal cancer. Br J Cancer 1998;78:511-514.

115. Gadgeel SM, Shields AF, Heilbrun LK, et al. Phase II study of paclitaxel and carboplatin in patients with advanced gastric cancer. Am J Clin Oncol 2003;26:37-41.

116. Kim JY, Do YR, Park KU, et al. A multi-center phase Il study of docetaxel plus cisplatin as first-line therapy in patients with metastatic squamous cell esophageal cancer. Cancer Chemother Pharmacol 2010;66:31-36.

117. Ohtsu A, Shimada Y, Shirao K, et al. Randomized phase III trial of fluorouracil alone versus fluorouracil plus cisplatin versus uracil and tegafur plus mitomycin in patients with unresectable, advanced gastric cancer: the Japan Clinical Oncology Group Study (JCOG9205). J Clin Oncol 2003;21:54-59.

118. Hong YS, Song SY, Lee SI, et al. A phase II trial of capecitabine in previously untreated patients with advanced and/or metastatic gastric cancer. Ann Oncol 2004;15:1344-1347.

119. Albertsson M, Johansson B, Friesland S, et al. Phase II studies on docetaxel alone every third week, or weekly in combination with gemcitabine in patients with primary locally advanced, metastatic, or recurrent esophageal cancer. Med Oncol 2007;24:407-412.

120. Ajani JA, Ilson DH, Daugherty $\mathrm{K}$, et al. Activity of taxol in patients with squamous cell carcinoma and adenocarcinoma of the esophagus. J Natl Cancer Inst 1994;86:1086-1091.

121. Ilson DH, Wadleigh RG, Leichman LP, et al. Paclitaxel given by a weekly 1-h infusion in advanced esophageal cancer. Ann Oncol 2007; 18:898-902.

122. Ross $P$, Nicolson $M$, Cunningham $D$, et al. Prospective randomized trial comparing mitomycin, cisplatin, and protracted venous-infusion fluorouracil (PVI 5-FU) with epirubicin, cisplatin, and PVI 5-FU in advanced esophagogastric cancer. J Clin Oncol 2002;20:1996-2004.

123. Sumpter K, Harper-Wynne C, Cunningham D, et al. Report of two protocol planned interim analyses in a randomised multicentre phase III study comparing capecitabine with fluorouracil and oxaliplatin with cisplatin in patients with advanced oesophagogastric cancer receiving ECF. Br J Cancer 2005;92:1976-1983.

124. Cunningham D, Starling N, Rao S, et al. Capecitabine and oxaliplatin for advanced esophagogastric cancer. N Engl J Med 2008;358:36-46.

125. Fuchs CS, Tomasek J, Yong CJ, et al. Ramucirumab monotherapy for previously treated advanced gastric or gastro-oesophageal junction adenocarcinoma (REGARD): an international, randomised, multicentre, placebo-controlled, phase 3 trial. Lancet 2014;383:31-39.

126. Wilke $\mathrm{H}$, Muro K, Van Cutsem E, et al. Ramucirumab plus paclitaxel versus placebo plus paclitaxel in patients with previously treated advanced gastric or gastro-oesophageal junction adenocarcinoma (RAINBOW): a double-blind, randomised phase 3 trial. Lancet Oncol 2014;15:1224-1235

127. Le DT, Uram JN, Wang H, et al. PD-1 blockade in tumors with mismatchrepair deficiency. N Engl J Med 2015;372:2509-2520.

128. Hironaka S, Ueda S, Yasui H, et al. Randomized, open-label, phase III study comparing irinotecan with paclitaxel in patients with advanced gastric cancer without severe peritoneal metastasis after failure of prior combination chemotherapy using fluoropyrimidine plus platinum: WJOG 4007 trial. J Clin Oncol 2013;31:4438-4444.

129. Sym SJ, Hong J, Park J, et al. A randomized phase II study of biweekly irinotecan monotherapy or a combination of irinotecan plus 5 -fluorouracil/leucovorin (mFOLFIRI) in patients with metastatic gastric adenocarcinoma refractory to or progressive after first-line chemotherapy. Cancer Chemother Pharmacol 2013;71:481-488.

130. Fuchs CS, Moore MR, Harker G, et al. Phase III comparison of two irinotecan dosing regimens in second-line therapy of metastatic colorecta cancer. J Clin Oncol 2003;21:807-814.

131. Sym SJ, Ryu MH, Lee JL, et al. Salvage chemotherapy with biweekly irinotecan, plus 5-fluorouracil and leucovorin in patients with advanced gastric cancer previously treated with fluoropyrimidine, platinum, and taxane. Am J Clin Oncol 2008;31:151-156.

132. Assersohn L, Brown G, Cunningham D, et al. Phase II study of irinotecan and 5-fluorouracil/leucovorin in patients with primary refractory or relapsed advanced oesophageal and gastric carcinoma. Ann Oncol 2004; 15:64-69.

133. Maugeri-Saccà M, Pizzuti L, Sergi D, et al. FOLFIRI as a second-line therapy in patients with docetaxel-pretreated gastric cancer: a historical cohort. J Exp Clin Cancer Res 2013;32:67

134. Shitara K, Doi T, Dvorkin M, et al. Trifluridine/tipiracil versus placebo in patients with heavily pretreated metastatic gastric cancer (TAGS): a randomised, double-blind, placebo-controlled, phase 3 trial. Lancet Oncol 2018;19:1437-1448.

135. U.S. Food and Drug Administration. FDA approves Lonsurf for recurrent metastatic gastric and gastroesophageal junction adenocarcinoma. 2019. Available at: https://www.fda.gov/Drugs/InformationOnDrugs/ ApprovedDrugs/ucm632032.htm. Accessed March 6, 2019.

136. Bando H, Doi T, Muro K, et al. A multicenter phase II study of TAS-102 monotherapy in patients with pre-treated advanced gastric cancer (EPOC1201). Eur J Cancer 2016;62:46-53.

137. U.S. Food and Drug Administration. FDA approves trastuzumab (Herceptin) 2010. Available at: http://wayback.archive-it.org/7993/ 20170113081145/http://www.fda.gov/AboutFDA/CentersOffices/ Office ofMedicalProductsandTobacco/CDER/ucm230418.htm. Accessed February 21, 2019

138. U.S. Food and Drug Administration. Ramucirumab in combination with paclitaxel. 2014. Available at: http://wayback.archive-it.org/7993/ 20170111231651/http://www.fda.gov/Drugs/InformationOnDrugs/ ApprovedDrugs/ucm421930.htm. Accessed February 21, 2019.

139. U.S. Food and Drug Administration. Ramucirumab. 2014. Available at http://wayback.archive-it.org/7993/20170111231700/http://www.fda.gov/ Drugs/InformationOnDrugs/ApprovedDrugs/ucm394260.htm. Accessed February 21, 2019.

140. Le DT, Uram JN, Wang H, et al. PD-1 blockade in mismatch repair deficient non-colorectal gastrointestinal cancers. J Clin Oncol 2016; 34(4_suppl):195

141. Soularue É, Cohen R, Tournigand C, et al. Efficacy and safety of trastuzumab in combination with oxaliplatin and fluorouracil-based chemotherapy for patients with HER2-positive metastatic gastric and 
gastro-oesophageal junction adenocarcinoma patients: a retrospective study. Bull Cancer 2015;102:324-331.

142. Tabernero J, Ohtsu A, Muro K, et al. Exposure-response analyses of ramucirumab from two randomized, phase III trials of second-line treatment of advanced gastric or gastroesophageal junction cancer. Mol Cancer Ther 2017:16:2215-2222.

143. Fuchs CS, Shitara K, Di Bartolomeo M, et al. Ramucirumab with cisplatin and fluoropyrimidine as first-line therapy in patients with metastatic gastric or junctional adenocarcinoma (RAINFALL): a double-blind randomised, placebo-controlled, phase 3 trial. Lancet Oncol 2019;20: 420-435.

144. Muro K, Chung HC, Shankaran V, et al. Pembrolizumab for patients with PD-L1-positive advanced gastric cancer (KEYNOTE-012): a multicentre, open-label, phase $1 \mathrm{~b}$ trial. Lancet Oncol 2016; 17:717-726

145. Bang Y-J, Muro K, Fuchs CS, et al. KEYNOTE-059 cohort 2: safety and efficacy of pembrolizumab (pembro) plus 5-fluorouracil (5-FU) and cisplatin for first-line (1L) treatment of advanced gastric cancer. J Clin Oncol 2017;35(15_suppl):4012.

146. Wainberg ZA, Jalal S, Muro K, et al. KEYNOTE-059 update: efficacy and safety of pembrolizumab alone or in combination with chemotherapy in patients with advanced gastric or gastroesophageal (G/GEJ) cancer. Ann Oncol 2017;28(suppl_5):v605-v649.

147. Catenacci DV Wainberg Z, Fuchs CS, et al. KEYNOTE-059 cohort 3: safety and efficacy of pembrolizumab monotherapy for first-line treatment of patients (pts) with PD-L1-positive advanced gastric/ gastroesophageal (G/GEJ) cancer. Ann Oncol 2017;28:suppl_3.

148. Kato K, Shah MA, Enzinger P, et al. KEYNOTE-590: Phase III study of first-line chemotherapy with or without pembrolizumab for advanced esophageal cancer. Future Oncol 2019;15:1057-1066.

149. Shitara K, Özgüroğlu M, Bang Y-J, et al. Pembrolizumab versus paclitaxel for previously treated, advanced gastric or gastro-oesophageal junction cancer (KEYNOTE-061): a randomised, open-label, controlled, phase 3 trial. Lancet 2018;392:123-133.

150. Doi T, Piha-Paul SA, Jalal SI, et al. Safety and antitumor activity of the anti-programmed death-1 antibody pembrolizumab in patients with advanced esophageal carcinoma. J Clin Oncol 2018;36:61-67.

151. Shah MA, Kojima T, Hochhauser D, et al. Efficacy and safety of pembrolizumab for heavily pretreated patients with advanced, metastatic adenocarcinoma or squamous cell carcinoma of the esophagus: the phase 2 KEYNOTE-180 study [published online December 20, 2018]. JAMA Oncol. doi: 10.1001/jamaoncol.2018.5441

152. Meerten EV, van Rij C, Tesselaar ME, et al. Definitive concurrent chemoradiation (CRT) with weekly paclitaxel and carboplatin for patients (pts) with irresectable esophageal cancer: a phase II study. J Clin Oncol 2010;28(15_suppl):e14508

153. van Westreenen HL, Westerterp M, Bossuyt PMM et al. Systematic review of the staging performance of 18F-fluorodeoxyglucose positron emission tomography in esophageal cancer. J Clin Oncol 2004 22:3805-3812.

154. Munden RF, Macapinlac HA, Erasmus JJ. Esophageal cancer: the role of integrated CT-PET in initial staging and response assessment after preoperative therapy. J Thorac Imaging 2006;21:137-145.

155. Lou F, Sima CS, Adusumilli PS, et al. Esophageal cancer recurrence patterns and implications for surveillance. J Thorac Oncol 2013;8: 1558-1562.

156. Sudo K, Taketa T, Correa AM, et al. Locoregional failure rate after preoperative chemoradiation of esophageal adenocarcinoma and the outcomes of salvage strategies. J Clin Oncol 2013; 31:4306-4310.

157. Dorth JA, Pura JA, Palta M, et al. Patterns of recurrence after trimodality therapy for esophageal cancer. Cancer 2014;120:2099-2105.

158. Sudo K, Xiao L, Wadhwa R, et al. Importance of surveillance and success of salvage strategies after definitive chemoradiation in patients with esophageal cancer. J Clin Oncol 2014;32:3400-3405.

159. Taketa T, Sudo K, Correa AM, et al. Post-chemoradiation surgical pathology stage can customize the surveillance strategy in patients with esophageal adenocarcinoma. J Natl Compr Canc Netw 2014;12: 1139-1144

160. MacLeod CM, (ed), The clinical evaluation of chemotherapeutic agents in cancer. In: Evaluation of Chemotherapeutic Agents. New York: Columbia University Press 1949:199.

161. Oken MM, Creech RH, Tormey DC, et al. Toxicity and response criteria of the Eastern Cooperative Oncology Group. Am J Clin Oncol 1982;5 649-655.

162. Schag CC, Heinrich RL, Ganz PA. Karnofsky performance status revisited: reliability, validity, and guidelines. J Clin Oncol 1984;2:187-193. 


\section{Individual Disclosures for the NCCN Esophageal and Esophagogastric Junction Cancers Panel}

\begin{tabular}{|c|c|c|c|c|}
\hline Panel Member & $\begin{array}{l}\text { Clinical Research Support/Data Safety } \\
\text { Monitoring Board }\end{array}$ & $\begin{array}{l}\text { Scientific Advisory Boards, Consultant, or } \\
\text { Expert Witness }\end{array}$ & $\begin{array}{l}\text { Promotional Advisory Boards, Consultant, } \\
\text { or Speakers Bureau }\end{array}$ & Specialties \\
\hline Jaffer A. Ajani, MD & None & $\begin{array}{l}\text { Astellas Pharma US, Inc.; AstraZeneca } \\
\text { Pharmaceuticals LP; Bristol-Myers Squibb } \\
\text { Company; Celgene Corporation; Eli Lilly and } \\
\text { Company; Genentech, Inc.; Merck \& Co., Inc.; } \\
\text { Roche Laboratories, Inc.; and Taiho } \\
\text { Parmaceuticals Co., Ltd. }\end{array}$ & None & $\begin{array}{l}\text { Medical Oncology, and } \\
\text { Gastroenterology }\end{array}$ \\
\hline David J. Bentrem, MD, MS & None & None & None & Surgery/Surgical Oncology \\
\hline Joseph Chao, MD & $\begin{array}{l}\text { Brooklyn ImmunoTherapeutics; Merck \& Co., } \\
\text { Inc.; and Novonco Therapeutics, Inc. }\end{array}$ & $\begin{array}{l}\text { AstraZeneca Pharmaceuticals LP; Boston } \\
\text { Biomedical, Inc.; Daiichi Sankyo, Co.; Eli Lilly } \\
\text { and Company; Foundation Medicine; Merck \& } \\
\text { Co., Inc.; and Taiho Parmaceuticals Co., Ltd. }\end{array}$ & Merck \& Co., Inc. & Medical Oncology \\
\hline Carlos Corvera, MD & None & None & None & Radiotherapy/Radiation Oncology \\
\hline Thomas A. D'Amico, MD & None & Scanlan & None & Surgery/Surgical Oncology \\
\hline Prajnan Das MD, MS, MPH & None & Adlai Nortye & None & Radiotherapy/Radiation Oncology \\
\hline Crystal S. Denlinger, MD & $\begin{array}{l}\text { Agios, Inc.; Amgen Inc.; Array BioPharma, Inc.; } \\
\text { AstraZeneca Pharmaceuticals LP; BeiGene; } \\
\text { Bristol-Myers Squibb Company; Eli Lilly and } \\
\text { Company; Macrogenics, Inc.; Medlmmune } \\
\text { Inc.; and Merrimack Pharmaceuticals }\end{array}$ & $\begin{array}{l}\text { Bayer HealthCare; Bristol-Myers Squibb } \\
\text { Company; and Merck \& Co., Inc. }\end{array}$ & None & Medical Oncology \\
\hline Peter C. Enzinger, MD & None & $\begin{array}{l}\text { Astellas Pharma US, Inc.; Eli Lilly and } \\
\text { Company; Merck \& Co., Inc.; and Taiho } \\
\text { Parmaceuticals Co., Ltd. }\end{array}$ & Daiichi Sankyo, Co. & Medical Oncology \\
\hline Paul Fanta, MD & None & None & None & $\begin{array}{l}\text { Hematology/Hematology } \\
\text { Oncology, and Medical Oncology }\end{array}$ \\
\hline Farhood Farjah, MD & None & None & None & Surgery/Surgical Oncology \\
\hline Hans Gerdes, MD & None & None & None & $\begin{array}{l}\text { Gastroenterology, and Internal } \\
\text { Medicine }\end{array}$ \\
\hline Michael Gibson, MD, PhD & $\begin{array}{l}\text { Boehringer Ingelheim } \mathrm{GmbH} \text {, and } \\
\text { Medlmmune, Inc. }\end{array}$ & $\begin{array}{l}\text { Bristol-Myers Squibb Company; Eli Lilly and } \\
\text { Company; and Merck \& Co., Inc. }\end{array}$ & $\begin{array}{l}\text { Bristol-Myers Squibb Company, and Eli Lilly } \\
\text { and Company }\end{array}$ & Medical Oncology \\
\hline Robert E. Glasgow, MD & Domain Surgical, Inc., and OmniGuide & Domain Surgical, Inc., and OmniGuide & Domain Surgical, Inc., and OmniGuide & Surgery/Surgical Oncology \\
\hline James A. Hayman, MD, MBA & None & None & None & Radiotherapy/Radiation Oncology \\
\hline Steven Hochwald, MD & None & None & None & Surgery/Surgical Oncology \\
\hline Wayne L. Hofstetter, MD & None & None & None & Surgery/Surgical Oncology \\
\hline David $\mathrm{H}$. Ilson, MD, PhD & None & $\begin{array}{l}\text { AstraZeneca Pharmaceuticals LP; Bayer } \\
\text { HealthCare; Bristol-Myers Squibb Company; } \\
\text { Eli illly and Company; Merck \& Co., Inc.; Pfizer } \\
\text { Inc.; and Roche Laboratories, Inc. }\end{array}$ & $\begin{array}{l}\text { Eli Lilly and Company, and Taiho } \\
\text { Parmaceuticals Co., Ltd. }\end{array}$ & $\begin{array}{l}\text { Medical Oncology, and Internal } \\
\text { Medicine }\end{array}$ \\
\hline Dawn Jaroszewski, MD & Biomet, Inc. & Biomet, Inc. & None & Surgery/Surgical Oncology \\
\hline Kimberly L. Johung, MD, PhD & None & None & None & Radiotherapy/Radiation Oncology \\
\hline Rajesh N. Keswani, MD & None & None & Boston Scientific Corporation & $\begin{array}{l}\text { Gastroenterology, and Internal } \\
\text { Medicine }\end{array}$ \\
\hline Lawrence R. Kleinberg, MD & Novocure & None & Novocure & Radiotherapy/Radiation Oncology \\
\hline Stephen Leong, MD & $\begin{array}{l}\text { Bristol-Myers Squibb Company; Deciphera } \\
\text { Pharmaceuticals, Inc.; and Karyopharm } \\
\text { Therapeutics, Inc. }\end{array}$ & Eli Lilly and Company & None & Medical Oncology \\
\hline Quan P. Ly, MD & None & None & None & Surgery/Surgical Oncology \\
\hline Kristina A. Matkowskyj, MD, PhD & None & None & None & Pathology \\
\hline Michael McNamara, MD & None & None & None & Medical Oncology \\
\hline Mary F. Mulcahy, MD & None & None & None & $\begin{array}{l}\text { Hematology/Hematology } \\
\text { Oncology, and Medical Oncology }\end{array}$ \\
\hline Ravi K. Paluri, MD, MPH & None & Amgen Inc. & Ipsen & Gastroenterology \\
\hline Haeseong Park, MD, MPH & $\begin{array}{l}\text { Ambrx; Amgen Inc.; Array Biopharma Inc.; } \\
\text { AstraZeneca Pharmaceuticals LP; Bayer } \\
\text { HealthCare; BeiGene; Bristol-Myers Squibb } \\
\text { Company; Daiichi Sankyo Co.; Eli Lilly and } \\
\text { Company; EMD Serono, Inc.; Genentech, Inc.; } \\
\text { Gilead Sciences, Inc.; GlaxoSmithKline; Incyte } \\
\text { Corporation; MacroGenics, Inc. Medlmmune } \\
\text { Inc.; Medivation, Inc.; Merck \& Co., Inc..; } \\
\text { Novartis Pharmaceuticals Corporation; Pfizer } \\
\text { Inc.; Puma Biotechnology;Regeneron } \\
\text { Pharmaceuticals, Inc.; and Taiho } \\
\text { Parmaceuticals Co., Ltd. }\end{array}$ & None & None & $\begin{array}{l}\text { Medical Oncology, and Internal } \\
\text { Medicine }\end{array}$ \\
\hline Kyle A. Perry, MD & Endostim, Inc., and Torax Medical, Inc. & None & None & Surgery/Surgical Oncology \\
\hline Jose Pimiento, MD & None & Advocare & None & Surgery/Surgical Oncology \\
\hline George A. Poultsides, MD, MS & None & None & None & Surgery/Surgical Oncology \\
\hline Robert Roses, MD & None & None & None & Surgery/Surgical Oncology \\
\hline Vivian E. Strong, MD & None & None & None & Surgery/Surgical Oncology \\
\hline Georgia Wiesner, MD & None & None & None & Genetics \\
\hline Christopher G. Willett, MD & None & None & None & Radiotherapy/Radiation Oncology \\
\hline Cameron D. Wright, MD & None & None & None & Surgery/Surgical Oncology \\
\hline
\end{tabular}

The NCCN Guidelines Staff have no conflicts to disclose. 